\title{
SHELLABLE AND COHEN-MACAULAY PARTIALLY ORDERED SETS
}

\author{
BY
}

\author{
ANDERS BJÖRNER
}

\begin{abstract}
In this paper we study shellable posets (partially ordered sets), that is, finite posets such that the simplicial complex of chains is shellable. It is shown that all admissible lattices (including all finite semimodular and supersolvable lattices) and all bounded locally semimodular finite posets are shellable. A technique for labeling the edges of the Hasse diagram of certain lattices, due to R. Stanley, is generalized to posets and shown to imply shellability, while Stanley's main theorem on the Jordan-Hölder sequences of such labelings remains valid. Further, we show a number of ways in which shellable posets can be constructed from other shellable posets and complexes. These results give rise to several new examples of CohenMacaulay posets. For instance, the lattice of subgroups of a finite group $G$ is Cohen-Macaulay (in fact shellable) if and only if $G$ is supersolvable. Finally, it is shown that all the higher order complexes of a finite planar distributive lattice are shellable.
\end{abstract}

Introduction. A pure finite simplicial complex $\Delta$ is said to be shellable if its maximal faces can be ordered $F_{1}, F_{2}, \ldots, F_{n}$ in such a way that $F_{k} \cap\left(\cup_{i=1}^{k-1} F_{i}\right)$ is a nonempty union of maximal proper faces of $F_{k}$ for $k=2,3, \ldots, n$. It is known that a shellable complex $\Delta$ must be Cohen-Macaulay, that is, a certain commutative ring associated with $\Delta$ is a Cohen-Macaulay ring (see the appendix for details). The notion of shellability, which originated in polyhedral theory, is emerging as a useful concept also in combinatorics with applications in matroid theory and order theory.

In this paper we study shellable posets (partially ordered sets), that is, finite posets for which the order complex consisting of all chains $x_{1}<x_{2}<\cdots<x_{k}$ is shellable. The material is organized as follows.

After some preliminary remarks in $\S 1$, we discuss in $\$ 2$ a certain type of labeling of the edges of the Hasse diagram of finite posets. We call posets which admit such labeling lexicographically shellable, and we prove that lexicographically shellable posets are indeed shellable. In lexicographically shellable posets the Möbius function can be interpreted as counting certain distinctly labeled maximal chains. We elaborate somewhat on this principle, point out its natural connection with shellability, and exemplify its use.

Received by the editors March 12, 1979. Presented to the Society, March 22, 1978, at the Symposium on Relations Between Combinatorics and Other Parts of Mathematics held at Ohio State University.

AMS (MOS) subject classifications (1970). Primary 06A10, 06A20; Secondary 05B35, 20D30, 52A25, $57 \mathrm{C05}$.

Key words and phrases. Shellable complex, Cohen-Macaulay complex, poset, lattice, Möbius function, admissible lattice, supersolvable group, barycentric subdivision, convex polytope, locally semimodular poset, Hirsch conjecture, planar distributive lattice. 
In $\$ 3$ we show that all admissible lattices (including all finite semimodular and supersolvable lattices) are lexicographically shellable. That such lattices are Cohen-Macaulay was conjectured by $R$. Stanley. In particular, the lattice of subgroups of a finite group $G$ is Cohen-Macaulay if and only if $G$ is supersolvable.

In $\$ 4$ we show that (lexicographic) shellability is preserved under several of the common poset constructions. We consider rank-selected subposets, direct products, ordinal sums, cardinal powers and interval posets.

The fifth section contains a proof that shellability is preserved under barycentric subdivision of simplicial complexes. We also point out that face-lattices of convex polytopes are shellable.

In $\$ 6$ we prove that bounded locally semimodular finite posets are shellable. Also, a certain inequality, known in polyhedral theory as the Hirsch conjecture, is shown to hold for such posets.

The order complex of a finite poset $P$ is the first member of a family of associated simplicial complexes $\Delta_{k}(P), k=1,2, \ldots$ In $\$ 7$ we prove that all these higher order complexes $\Delta_{k}(P)$ are shellable when $P$ is a finite planar distributive lattice.

Finally, in an appendix we briefly review the definitions and basic properties of shellable and Cohen-Macaulay complexes.

The author wants to thank R. Stanley, whose work inspired these investigations, for many stimulating discussions. Thanks are due also to K. Baclawski, A. Garsia, V. Klee and S. Provan for helpful comments.

1. Preliminaries. For poset terminology not otherwise explained we refer to [2]. A poset is said to be bounded if it has a least element and a greatest element. These will always be denoted by $\hat{0}$ and $\hat{1}$ respectively. For a bounded poset $P$ we let $\bar{P}$ denote the subposet $P-\{\hat{0}, \hat{1}\}$. For a poset $Q$ we let $\hat{Q}$ denote the (essentially) unique bounded poset $P$ such that $\bar{P}=Q$. A finite poset is said to be pure if all maximal chains have the same length. A pure poset satisfies the Jordan-Dedekind condition: all unrefinable chains between two comparable elements have the same length. We shall call a poset graded if it is finite, bounded and pure. Any element $x$ of a graded poset $P$ has a well-defined rank $\rho(x)$ equal to the common length of all unrefinable chains from $\hat{0}$ to $x$ in $P$. The symbol " $\prec$ " denotes the covering relation: $x<y$ means that $x<y$ and $x<z<y$ for no $z$. The notation "c: $x_{0}<x_{1}<\cdots<x_{n}$ " will be frequently used for denoting a chain $\mathrm{c}=$ $\left\{x_{0}, x_{1}, \ldots, x_{n}\right\}$ such that $x_{0}<x_{1}<\cdots<x_{n}$.

The order complex $\Delta(P)$ of a finite poset $P$ is the simplicial complex of all chains of $P$. We shall say that a finite pure poset $P$ is shellable if its order complex $\Delta(P)$ is shellable. Similarly, $P$ will be called Cohen-Macaulay if $\Delta(P)$ is a Cohen-Macaulay complex. These properties of simplicial complexes are defined and commented on in an appendix at the end of this paper. Note in particular that a shellable poset must be Cohen-Macaulay, and that a Cohen-Macaulay poset must be pure. For convenience, when we write simply "Cohen-Macaulay" (for instance, in Theorem 3.3) the intention is that any one of the slightly different concepts of "CohenMacaulay-ness" defined in the appendix may be applied. Note that a finite poset $P$ 
is shellable (Cohen-Macaulay) if and only if $\hat{\boldsymbol{P}}$ is shellable (Cohen-Macaulay).

The cardinality of a finite set $S$ will be written $|S|$. For a positive integer $n$ we let [n] denote the set $\{1,2, \ldots, n\}$.

2. Lexicographically shellable posets. For any finite poset $P$ we let $C(P)$ denote its covering relation, $C(P)=\{(x, y) \in P \times P \mid x<y\}$. An edge-labeling of $P$ is a map $\lambda: C(P) \rightarrow \Lambda$, where $\Lambda$ is some poset. An edge-labeling therefore corresponds to an assignment of elements of $\Lambda$ to the edges of the Hasse diagram of $P$. An unrefinable chain $x_{0} \prec x_{1} \prec \cdots \prec x_{n}$ in a poset with an edge-labeling $\lambda$ will be called rising if $\lambda\left(x_{0}, x_{1}\right)<\lambda\left(x_{1}, x_{2}\right)<\cdots<\lambda\left(x_{n-1}, x_{n}\right)$.

Definition 2.1. Let $\lambda: C(P) \rightarrow \Lambda$ be an edge-labeling of a graded poset $P$. $\lambda$ is said to be an $R$-labeling if in every interval $[x, y]$ of $P$ there is a unique rising unrefinable chain $x=x_{0} \prec x_{1} \prec \cdots \prec x_{n}=y . \lambda$ is said to be an L-labeling in case (i) $\lambda$ is an $R$-labeling and (ii) for every interval $[x, y]$ of $P$ if $x=x_{0} \prec x_{1}$ $\prec \cdots \prec x_{n}=y$ is the unique rising unrefinable chain and $x<z<y, z \neq x_{1}$, then $\lambda\left(x, x_{1}\right)<\lambda(x, z)$.

Definition 2.2. A poset is lexicographically shellable (or L-shellable) if it is graded and admits an $L$-labeling.

The following result is fundamental for this paper.

Theorem 2.3. Let $P$ be a lexicographically shellable poset. Then $P$ is shellable.

The proof will be given after some useful technical properties of $L$-labelings have been derived.

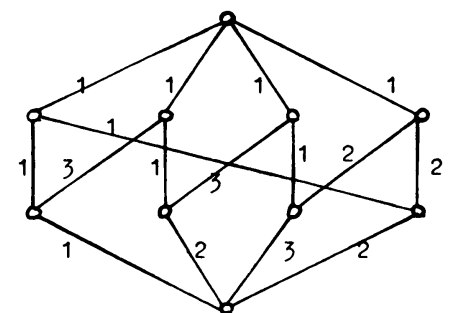

(a)

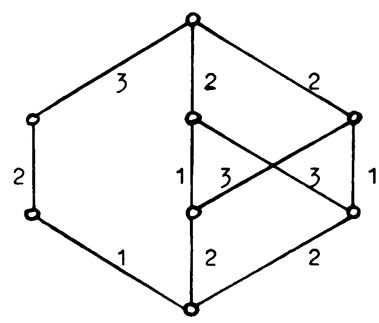

(c)

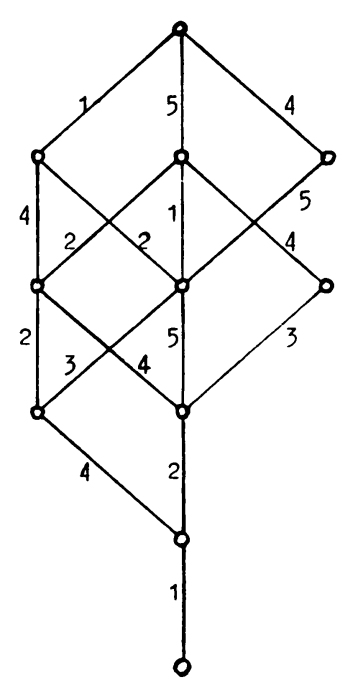

(b) 
EXAMPLE 2.4. To illustrate the above definitions we exhibit $L$-labelings $(\Lambda=Z)$ of the face-lattice of a square (Figure 1a) and of the poset of partitions of the integer 6 ordered by refinement (Figure 1b). The poset $P$ of Figure 1c has been given an $R$-labeling which is not an $L$-labeling. In fact, by the above theorem there cannot exist any $L$-labeling of $P$, since $P$ is clearly not shellable.

With every unrefinable $n$-chain c: $x_{0} \prec x_{1} \prec \cdots \prec x_{n}$ of a poset $P$ having an edge-labeling $\lambda: C(P) \rightarrow \Lambda$ we associate the $n$-tuple

$$
\pi(\mathbf{c})=\left(\lambda\left(x_{0}, x_{1}\right), \lambda\left(x_{1}, x_{2}\right), \ldots, \lambda\left(x_{n-1}, x_{n}\right)\right) \in \Lambda^{n} .
$$

Following Stanley [19] we call $\pi(c)$ the Jordan-Hölder sequence of c. For any poset $\Lambda$ the cartesian product $\Lambda^{n}$ is partially ordered by the lexicographic order: $\left(a_{1}, a_{2}, \ldots, a_{n}\right) \in \Lambda^{n}$ precedes $\left(b_{1}, b_{2}, \ldots, b_{n}\right) \in \Lambda^{n}$ if and only if $a_{i}<b_{i}$ in the first coordinate where they differ.

When dealing with a poset $P$ having an $R$-labeling we shall for the rest of this paper let $c(x, y)$ denote the unique rising unrefinable chain from $x$ to $y$ in $P$. The Jordan-Hölder sequences of $L$-labelings have the following important property.

Proposition 2.5. Let $\lambda: C(P) \rightarrow \Lambda$ be an $R$-labeling of a graded poset $P$. Then (i) and (ii) below are equivalent conditions, and both are implied by (iii):

(i) $\lambda$ is an L-labeling,

(ii) for every interval $[x, y]$ in $P$ and every unrefinable chain d from $x$ to $y$ other than $\mathbf{c}(x, y)$ it is true that $\pi(\mathbf{c}(x, y))<\pi(\mathbf{d})$ in the lexicographic order,

(iii) for every interval $[x, y]$ in $P$, if $x \prec x_{1}$ is the first link of $\mathrm{c}(x, y): x=x_{0} \prec x_{1}$ $\prec \cdots \prec x_{n}=y$ then $\lambda\left(x, x_{1}\right) \leqslant \lambda(u, v)$ for all $x \leqslant u<v<y$.

Proof. (i) $\Rightarrow$ (ii): Let d: $x=y_{0} \prec y_{1} \prec \cdots \prec y_{n}=y$ be an unrefinable chain different from $c(x, y): x=x_{0} \prec x_{1} \prec \cdots \prec x_{n}=y$. If $k$ is the least index such that $x_{k} \neq y_{k}$, we know that $\lambda\left(x_{k-1}, x_{k}\right)<\lambda\left(x_{k-1}, y_{k}\right)=\lambda\left(y_{k-1}, y_{k}\right)$, since $\lambda$ is an $L$-labeling and $x_{k-1} \prec x_{k} \prec \cdots \prec x_{n}=y$ must be the unique rising chain of the interval $\left[x_{k-1}, y\right]$. Hence, $\pi(\mathbf{c}(x, y))<\pi(d)$.

(ii) $\Rightarrow$ (i): Let $x \prec z \leqslant y, z \neq x_{1}$, where $\mathrm{c}(x, y): x=x_{0} \prec x_{1} \prec \cdots<x_{n}=y$. Also, let $\mathrm{c}(z, y): z=z_{0} \prec z_{1} \prec \cdots \prec z_{n-1}=y$. Now, $\pi(\mathbf{c}(x, y))<\pi(\mathbf{d})$, where d: $x \prec z_{0} \prec z_{1} \prec \cdots \prec z_{n-1}=y$. Hence, $\lambda\left(x, x_{1}\right) \leqslant \lambda\left(x, z_{0}\right)$. If $\lambda\left(x, x_{1}\right)=\lambda\left(x, z_{0}\right)$ then $\lambda\left(x, z_{0}\right)=\lambda\left(x, x_{1}\right) \leqslant \lambda\left(x_{1}, x_{2}\right) \leqslant \lambda\left(z_{0}, z_{1}\right) \leqslant \lambda\left(z_{1}, z_{2}\right)<\cdots<\lambda\left(z_{n-2}, y\right)$ so that $\mathrm{d}$ is also rising from $x$ to $y$, which contradicts the uniqueness of $\mathrm{c}(x, y)$. Consequently, $\lambda\left(x, x_{1}\right)<\lambda(x, z)$.

(iii) $\Rightarrow$ (i): Preserve the meaning of $x, y, z, \mathrm{c}(x, y), \mathrm{c}(z, y)$ and $\mathrm{d}$ from the preceding paragraph. By assumption, $\lambda\left(x, x_{1}\right) \leqslant \lambda(x, z)$ and $\lambda\left(x, x_{1}\right)<\lambda\left(z, z_{1}\right)$. Hence, if $\lambda\left(x, x_{1}\right)=\lambda(x, z)$ then $\mathrm{d}$ would be rising. So, $\lambda\left(x, x_{1}\right)<\lambda(x, z)$.

(i) does not imply (iii) as simple counterexamples show.

Let us say that a sequence $\pi=\left(a_{1}, a_{2}, \ldots, a_{n}\right)$ of elements from a poset $\Lambda$ has a descent at $e, e \in[n-1]$, if $a_{e} \$ a_{e+1}$. The sequence $\pi$ is said to have descent set $D(\pi)=\left\{e \in[n-1] \mid a_{e} \nless a_{e+1}\right\}$. 
LemMA 2.6. Let $\lambda: C(P) \rightarrow \Lambda$ be an $L$-labeling of a graded poset $P$ of length $n$, and suppose that $\mathbf{m}: \hat{0}=x_{0} \prec x_{1} \prec \cdots \prec x_{n}=\hat{1}$ is a maximal chain of $P$. Then $\pi(\mathrm{m})$ has $a$ descent at $e \in[n-1]$ if and only if $\mathbf{h} \cap \mathbf{m}=\mathbf{m}-\left\{x_{e}\right\}$ for some maximal chain $\mathbf{h}$ such that $\pi(\mathbf{h})<\pi(\mathbf{m})$ in the lexicographic order on $\Lambda^{n}$.

Proof. Let $\mathrm{c}\left(x_{e-1}, x_{e+1}\right): x_{e-1} \prec y \prec x_{e+1}$ be the unique rising chain from $x_{e-1}$ to $x_{e+1}$ and define d: $\hat{0}=x_{0} \prec x_{1} \prec \cdots \prec x_{e-1} \prec y \prec x_{e+1} \prec x_{e+2} \prec \cdots \prec$ $x_{n}=\hat{1}$. If $\pi(\mathrm{m})$ has a descent at $e$, then $y \neq x_{e}$ and $\mathbf{h}=\mathrm{d}$ has the required properties. Conversely, if $\pi(\mathrm{m})$ does not have a descent at $e$, then $\mathbf{m}=\mathbf{d}$ and since $\lambda$ is an $L$-labeling it follows that $\pi(\mathbf{m})<\pi(\mathbf{h})$ for all maximal chains $\mathbf{h}$ such that $\mathbf{h} \cap \mathbf{m}=\mathbf{m}-\left\{x_{e}\right\}$.

Proof of Theorem 2.3. Let $\lambda: C(P) \rightarrow \Lambda$ be an $L$-labeling of the graded poset $P$. We must prove that the set $\mathfrak{N}$ of maximal chains of $P$ has a shelling, that is, a linear order $\Omega$ such that

if $\mathbf{k}<^{\mathbf{\Omega}} \mathbf{m}$ for $\mathbf{k}, \mathbf{m} \in \mathscr{T}$ then there is an $\mathbf{h} \in \mathscr{T}$ with $\mathbf{h}<^{\mathbf{\Omega}} \mathbf{m}$ such that $(\mathbf{k} \cap \mathbf{m}) \subseteq(\mathbf{h} \cap \mathbf{m})$ and $|\mathbf{h} \cap \mathbf{m}|=|\mathbf{m}|-1$.

We shall show that, in fact, every linear order of the set $\mathfrak{N}$ that is compatible with the lexicographic order of the associated Jordan-Hölder sequences is a shelling.

Assign a linear order $\Omega$ to the set $\mathscr{T}$ in such a way that if $\pi(m)<\pi\left(m^{\prime}\right)$ in the lexicographic order on $\Lambda^{n}$ then $\mathbf{m}<^{\Omega} \mathbf{m}^{\prime}$. This is clearly always possible. Now, consider two maximal chains of $P \mathbf{k}: \hat{\mathbf{0}}=y_{0} \prec y_{1} \prec \cdots \prec y_{n}=\hat{\mathbf{1}}$ and $\mathbf{m}: \hat{\mathbf{0}}=x_{0}$ $\prec x_{1} \prec \cdots \prec x_{n}=\hat{1}$, and suppose that $\mathbf{k}<^{\Omega} \mathbf{m}$. Let $d$ be the greatest integer such that $x_{i}=y_{i}$ for $i=0,1, \ldots, d$, and let $g$ be the least integer greater than $d$ such that $x_{g}=y_{g}$. Then $g-d \geqslant 2$ and $d<i<g$ implies that $x_{i} \neq y_{i}$. The chain $x_{d} \prec x_{d+1} \prec \cdots \prec x_{g}$ cannot be the unique rising chain in the interval $\left[x_{d}, x_{g}\right]$, because in that case $\pi(\mathbf{m})$ would precede $\pi(\mathbf{k})$ in the lexicographic order, which contradicts $\mathbf{k}<^{\Omega} \mathbf{m}$. Therefore, $\pi(\mathbf{m})$ must have a descent at some $e$ such that $d<e<g$. Consequently, by Lemma 2.6 there is a $\mathbf{h} \in \mathfrak{N}$ with $\pi(\mathbf{h})<\pi(\mathbf{m})$, hence $\mathbf{h}<\mathbf{\Omega} \mathbf{m}$, such that $\mathbf{h} \cap \mathbf{m}=\mathbf{m}-\left\{x_{e}\right\} \supseteq \mathbf{k} \cap \mathbf{m}$. This completes the proof.

Let $P$ be a graded poset of length $n$. Then $\rho(x) \in[n-1]$ for all $x \in \bar{P}$. For any subset $S \subseteq[n-1]$ we define the rank-selected subposet $P_{S}$ by

$$
P_{S}=\{x \in P \mid \rho(x) \in S \cup\{0, n\}\} .
$$

Thus, $P_{S}$ is also graded and its length equals $|S|+1$. For the definition and fundamental properties of the Möbius function $\mu_{Q}$ of a finite poset $Q$ we refer to [17]. It will be convenient to write $\mu(Q)$ instead of $\mu_{Q}(\hat{0}, \hat{1})$ for a finite bounded poset $Q$.

TheOREM 2.7 (R. STANLEY). Let $P$ be a graded poset of length $n$, and suppose that an $R$-labeling of $P$ is given. If $S \subseteq[n-1]$, then $(-1)^{|S|+1} \mu\left(P_{S}\right)$ is equal to the number of maximal chains $\mathbf{m}$ in $P$ with Jordan-Hölder sequences $\pi(\mathbf{m})$ having descent set $D(\pi(\mathbf{m}))=S$. 
This theorem, formulated for admissible lattices, plays a fundamental role in [18] and [19]. The proof below is modelled on Stanley's proof of Theorem 1.2 in [18, p. 206].

Proof. Let $\mathfrak{T}$ denote the set of maximal chains of $P$, and similarly for $S \subseteq[n-1]$ let $\Re_{S}$ be the set of maximal chains of $P_{S}$. The $R$-labeling of $P$ determines a map $\theta: \mathfrak{N}_{s} \rightarrow \mathfrak{N}$ defined by "filling in the gaps with rising chains". More precisely, if $\mathrm{m} \in \mathfrak{N}_{s}$, m: $\hat{0}<x_{i_{1}}<x_{i_{2}}<\cdots<x_{i_{s}}<\hat{\mathbf{1}}$ where $s=|S|$, $\rho\left(x_{i_{k}}\right)=i_{k}$ and $i_{k} \in S$ for $k=1,2, \ldots, s$, then $\theta(\mathrm{m})=$ $\mathbf{c}\left(\hat{0}, x_{i_{1}}\right) * \mathbf{c}\left(x_{i_{1}}, x_{i_{2}}\right) * \cdots * \mathbf{c}\left(x_{i_{s}}, \hat{1}\right)$, where "*" denotes the operation of concatenating chains. We claim that $\theta$ is a bijection between $\mathfrak{T}_{s}$ and the set $\{m \in \mathfrak{N} \mid D(\pi(\mathbf{m})) \subseteq S\}$. By construction, $D(\pi(\theta(\mathbf{m}))) \subseteq S$ for all $\mathbf{m} \in \mathfrak{N}_{S}$. Since $\mathbf{m}=\theta(\mathbf{m}) \cap P_{S}, \theta$ is injective. Since for every $\mathbf{m}^{\prime} \in\{\mathbf{m} \in \Re \mid D(\pi(\mathbf{m})) \subseteq S\}$ it is true that $\mathbf{m}^{\prime}=\theta\left(\mathbf{m}^{\prime} \cap P_{S}\right), \theta$ is surjective onto that set.

Now, let $\delta(S)$ be the number of maximal chains $m \in \Re$ such that $D(\pi(m))=$ $S$. We have just proved that $\left|\Re_{S}\right|=\Sigma_{T \subseteq S} \delta(T)$. Therefore,

$\delta(S)=\sum_{T \subseteq S}(-1)^{|S-T|}\left|\mathscr{R}_{T}\right|=(-1)^{|S|+1} \sum_{T \subseteq S}(-1)^{|T|+1}\left|\mathscr{R}_{T}\right|=(-1)^{|S|+1} \mu\left(P_{S}\right)$,

where the first equality is obtained by Möbius inversion [17, p. 344] and the last is provided by the well-known theorem of P. Hall's [17, Proposition 6, p. 346].

Let $P$ be a graded poset of length $n$ and suppose that there is an $L$-labeling $\lambda$ : $C(P) \rightarrow \Lambda$ of $P$. We know from Theorem 2.3 that $P$ is shellable and from Theorem 2.7 that $(-1)^{n} \mu(P)$ equals the number of maximal chains $x_{0} \prec x_{1} \prec \cdots \prec x_{n}$ of $P$ such that

$$
\lambda\left(x_{0}, x_{1}\right) \nless \lambda\left(x_{1}, x_{2}\right) \nless \cdots \nless \lambda\left(x_{n-1}, x_{n}\right) .
$$

Judging from the above proofs these results may appear totally unrelated. There is, however, a close connection. As we shall informally indicate, this connection can be seen as a special case of a more widely applicable principle.

Let $\Delta$ be a shellable simplicial complex and let $F_{1}, F_{2}, \ldots, F_{t}$ be a shelling of $\Delta$ (cf. the appendix). Each facet $F_{j}$ then has a unique minimal face $\dot{F}_{j}$ that is not contained in $F_{i}$ for $i<j$. It is easy to see that

(a) $x \in \stackrel{\circ}{F}_{j}$ if and only if $F_{j}-\{x\} \subseteq F_{i}$ for some $i<j$. Also, using the elementary formula $\tilde{\chi}(A)+\tilde{\chi}(B)=\tilde{\chi}(A \cup B)+\tilde{\chi}(A \cap B)$, it becomes immediately clear that

$(\beta)$ the number of facets $F_{j}$ such that $\stackrel{\circ}{F}_{j}=F_{j}$ equals $(-1)^{\operatorname{dim} \Delta} \tilde{\chi}(\Delta)$, where $\tilde{\chi}$ denotes the Euler characteristic in reduced simplicial homology.

After these general considerations, let us return to the graded poset $\boldsymbol{P}$ with its $L$-labeling $\lambda$. In the proof of Theorem 2.3 we showed how to derive from $\lambda$ a shelling of the order complex $\Delta(P)$. If $\hat{0}$ and $\hat{1}$ are removed from the maximal chains of $P$ we obtain a shelling of $\Delta(\bar{P})$. For any maximal chain $\mathbf{m}$ of $\bar{P}$, Lemma 2.6 tells us, in view of statement $(\alpha)$ above, precisely that $\dot{m}=\mathbf{m} \cap P_{s}$, where $S=D(\pi(\hat{\mathbf{m}}))$ and $\hat{\mathbf{m}}=\mathbf{m} \cup\{\hat{0}, \hat{\mathbf{1}}\}$. Therefore, the facets $\mathbf{m}$ of $\Delta(\bar{P})$ such that $\stackrel{\circ}{\mathbf{m}}=\mathbf{m}$ are those maximal chains $\mathbf{m}$ of $\bar{P}$ such that $D(\pi(\hat{\mathbf{m}}))=[n-1]$, or, in other words, such that $\hat{\mathbf{m}}: x_{0} \prec x_{1} \prec \cdots \prec x_{n}$ satisfies $(*)$. Since $\mu(P)=\tilde{\chi}(\Delta(\bar{P}))$ by $\mathrm{P}$. 
Hall's theorem [17, Proposition 6, p. 346], we find that statement $(\beta)$ can be rephrased, for our particular shelling, as saying that the number of maximal chains of $P$ which satisfy (*) equals $(-1)^{n} \mu(P)$.

The considerations of the preceding paragraph can be fully generalized to rank-selected subposets $P_{S}$ of $P$. It will later be proved (Theorem 4.1) that any shelling of $P$ induces shellings of all $P_{S}, S \subseteq[n-1]$. Still assuming that we have an $L$-labeling of $P$, it can be shown that the conclusion of Theorem 2.7 is just a reformulation of statement $(\beta)$ above for the shelling which is induced on $\bar{P}_{S}$ by the lexicographic shelling of $\boldsymbol{P}$. We omit further details.

In Examples 2.9 and 3.8 below we present some interesting applications of the following general principle.

Proposition 2.8. Let $P$ be a graded poset and $\lambda: C(P) \rightarrow \Lambda$ an L-labeling of $P$. Suppose that $Q$ is a subset of $P$ such that

(i) $\hat{0}, \hat{\imath} \in Q$, and

(ii) if $x, y \in Q$ and $x<y$ in $P$ then $\mathrm{c}(x, y) \subseteq Q$, where $\mathrm{c}(x, y)$ is the unique rising chain from $x$ to $y$ in $P$.

Then $Q$ is a graded poset under the inherited order, $C(Q) \subseteq C(P)$, and the restriction of $\lambda$ to $C(Q)$ is an L-labeling of $Q$.

Proof. The verification is straightforward.

EXAMPLE 2.9. Let $\Pi_{n}$ denote the lattice of partitions of the set [n] ordered by refinement. A covering relation $\pi \prec \pi^{\prime}$ in $\Pi_{n}$ corresponds to a merging of two distinct blocks $B_{1}$ and $B_{2}$ of $\pi$ into one block $B_{1} \cup B_{2}$ of $\pi^{\prime}$. Let $\lambda\left(\pi \prec \pi^{\prime}\right)=$ $\max \left\{\min B_{1}, \min B_{2}\right\}$. It is not hard to see that this edge-labeling $\lambda$ of $\Pi_{n}$ is an $L$-labeling. $\lambda$ was first suggested to the author by $I$. Gessel. $\lambda$ can also be obtained as the induced edge-labeling of an admissible map by the standard construction for supersolvable lattices (cf. the following section and [19, Proposition 2.4, p. 363]). The Jordan-Hölder sequences of maximal chains of $\Pi_{n}$ under the labeling $\lambda$ are permutations of the set $\{2,3, \ldots, n\}$. It is an easy exercise to verify that there are $(n-1)$ ! maximal chains with strictly decreasing Jordan-Hölder sequences. Hence, by Theorem 2.7, $\mu\left(\Pi_{n}\right)=(-1)^{n-1}(n-1)$ !.

Following G. Kreweras [10] we shall call a partition $\pi \in \Pi_{n}$ noncrossing if for any blocks $B_{1}$ and $B_{2}$ of $\pi$ the conditions $x_{1}, x_{3} \in B_{1}, x_{2}, x_{4} \in B_{2}$ and $x_{1}<x_{2}<$ $x_{3}<x_{4}$ imply $B_{1}=B_{2}$. It was observed jointly by P. Edelman and the author that the set $\mathcal{T}_{n}$ of all noncrossing partitions of $[n]$ satisfies condition 2.8(i) and (ii) above. Hence, $\lambda$ restricts to an $L$-labeling of $\mathscr{T}_{n}$, which in fact is a lattice under the inherited refinement order [10, Théorèmes 2 et 3, p. 335]. Consequently, $\sigma_{n}$ is a shellable lattice. Let us now count the number of maximal chains in $\mathcal{T}_{n}$ which have strictly decreasing Jordan-Hölder sequences.

Suppose that we select $n$ elements $x_{1}, x_{2}, \ldots, x_{n}$ from a set in which a nonassociative, noncommutative binary composition is defined. A correct bracketing of the sequence $x_{1} x_{2} \ldots x_{n}$ is an assignment of left and right bracket symbols "(" and ")" to the sequence in such a way that the resulting expression is well-formed with respect to the binary composition. Given a correct bracketing $\beta$ of the sequence 
$x_{1} x_{2} \ldots x_{n}$ we define a maximal chain $f(\beta): \pi_{0} \prec \pi_{1} \prec \cdots \prec \pi_{n-1}$ in $\Pi_{n}$ by the following rule:

(1) $\pi_{0}$ is the discrete partition with only singleton blocks,

(2) $\pi_{i+1}$ is obtained from $\pi_{i}$ by joining the block to which $n-i$ belongs with the block to which $\varphi(n-i)$ belongs, where $\varphi:([n]-\{1\}) \rightarrow[n]$ is defined as follows:

(i) If there are no brackets or only left brackets between $x_{k-1}$ and $x_{k}$ then $\varphi(k)=k-1$.

(ii) If there are right brackets between $x_{k-1}$ and $x_{k}$, let $)^{\prime}$ be that right bracket which stands closest to $x_{k}$ and let (' be the left "mate" of )' (in the obvious sense). Then $\varphi(k)$ is that integer for which $x_{\varphi(k)}$ is the first element to the right of ('.

For example, if $\beta$ is the correct bracketing

$$
\beta:\left(x_{1} x_{2}\right)\left(\left(x_{3}\left(x_{4} x_{5}\right)\right) x_{6}\right),
$$

then $f(\beta)$ is the maximal chain $\pi_{0} \prec \pi_{1} \prec \cdots \prec \pi_{5}$ in $\Pi_{6}$ given by

$$
\begin{array}{r}
\pi_{0}: 1-2-3-4-5-6, \\
\pi_{1}: 1-2-36-4-5, \\
\pi_{2}: 1-2-36-45, \\
\pi_{3}: 1-2-3456, \\
\pi_{4}: 13456-2, \\
\pi_{5}: 123456,
\end{array}
$$

and the Jordan-Hölder sequence of $f(\beta)$ under $\lambda$ is $(6,5,4,3,2)$. It is not hard to prove for the general case that the elements of $f(\beta)$ have to be noncrossing partitions and that the Jordan-Hölder sequence of $f(\beta)$ must be strictly decreasing. In fact, one can show that $f$ defines a bijection between the set of correct bracketings of the sequence $x_{1} x_{2} \ldots x_{n}$ and the set of maximal chains of $\mathcal{T}_{n}$ with strictly decreasing Jordan-Hölder sequences. It is well known in combinatorics that the number of correct bracketings of $x_{1} x_{2} \ldots x_{n}$ is equal to the Catalan number

$$
C_{n}=\frac{1}{n}\left(\begin{array}{c}
2 n-2 \\
n-1
\end{array}\right)
$$

Hence, we find using Theorem 2.7 that

$$
\mu\left(\mathscr{T}_{n}\right)=(-1)^{n-1} \frac{1}{n}\left(\begin{array}{c}
2 n-2 \\
n-1
\end{array}\right)
$$

This formula was first obtained by Kreweras [10, Théorème 6, p. 348].

3. Admissible lattices. Let $L$ be a finite lattice and $\omega: I(L) \rightarrow \mathbf{P}$ a map from the set $I(L)$ of join-irreducibles of $L$ to the set $\mathbf{P}$ of positive integers. Such a map $\omega$ induces an edge-labeling $\gamma: C(L) \rightarrow \mathbf{P}$ of $L$ by the rule

$$
\gamma(x \prec y)=\min \{\omega(z) \mid z \in I(L), x<x \vee z=y\}
$$

If $\gamma$ is an $R$-labeling then $\omega$ is called an admissible map. A finite lattice $L$ is said to be admissible if it is graded and there exists an admissible map $\omega: I(L) \rightarrow$ P. These definitions are due to R. Stanley [19], who also showed that all upper-semimodular 
and all supersolvable finite lattices are admissible. The class of admissible lattices is larger, but no other major subclass of interest seems to be known.

TheOREM 3.1. An admissible lattice is lexicographically shellable.

Proof. Let $\omega: I(L) \rightarrow \mathbf{P}$ be an admissible map of a graded lattice $L$. To show that the induced edge-labeling $\gamma$ is an $L$-labeling it will suffice to verify condition (iii) of Proposition 2.5 for $\lambda=\gamma$. Assume that $\mathrm{c}(x, y): x=x_{0} \prec x_{1} \prec \cdots \prec x_{n}=$ $y$ is the unique maximal chain in the interval $[x, y]$ which is rising under the $R$-labeling $\gamma$. Let $I^{\prime}=\{z \in I(L) \mid x<x \vee z<y\}, a=\min \left\{\omega(z) \mid z \in I^{\prime}\right\}$ and $\omega\left(z^{\prime}\right)$ $=a, z^{\prime} \in I^{\prime}$. Clearly, $a \leqslant \gamma(u, v)$ for all $x<u<v<y$. Let $i$ be the least integer such that $z^{\prime} \leqslant x_{i}$. Then $a \leqslant \gamma\left(x, x_{1}\right) \leqslant \gamma\left(x_{i-1}, x_{i}\right)=a$. Hence, $\gamma\left(x, x_{1}\right)=a<$ $\gamma(u, v)$ for all $x \leqslant u<v \leqslant y$.

Corollary 3.2. An admissible lattice is Cohen-Macaulay.

Corollary 3.2 was conjectured by R. Stanley in [20, p. 60]. It was previously known that finite upper-semimodular lattices are Cohen-Macaulay and that finite distributive lattices are shellable. These results are due to J. Folkman [7] and S. Provan [12] respectively.

Our results contain the remaining pieces of information needed to fully settle the question: for which finite groups $G$ is the lattice of subgroups $L(G)$ CohenMacaulay? The Cohen-Macaulay property has been studied for certain subposets of $L(G)$ by D. Quillen [14].

THEOREM 3.3. Let $G$ be a finite group and $L(G)$ its lattice of subgroups. Then the following conditions are equivalent:

(i) $G$ is supersolvable,

(ii) $L(G)$ is Cohen-Macaulay,

(iii) $L(G)$ is lexicographically shellable.

Proof. We have the following loop of implications: $G$ is supersolvable ${ }^{A} \Rightarrow$ $L(G)$ is admissible ${ }^{\mathrm{B}} \Rightarrow L(G)$ is lexicographically shellable ${ }^{\mathrm{C}} \Rightarrow L(G)$ is shellable $\mathrm{D} \Rightarrow L(G)$ is Cohen-Macaulay $\mathrm{E} \Rightarrow L(G)$ is pure $\mathrm{F} \Rightarrow G$ is supersolvable. A is due to R. Stanley ([18, Example 2.5, p. 204] and [19, Proposition 2.4, p. 363]). B and C are provided by Theorems 3.1 and 2.3 respectively. $D$ and $E$ are well known (cf. the appendix). F was proved by K. Iwasawa in 1941 (see [22, Theorem 9, p. 9] or [2, Theorem 21, p. 177]).

Some of the important examples of $L$-shellable posets admit $L$-labelings with the following stronger property.

Definition 3.4. An $S L$-labeling $\lambda$ of a graded poset $P$ is an $L$-labeling $\lambda$ : $C(P) \rightarrow \Lambda$ such that if $x=x_{0} \prec x_{1} \prec \cdots \prec x_{n}=y$ is the unique rising maximal chain in the interval $[x, y]$ then $\lambda\left(x_{n-1}, y\right)>\lambda(z, y)$ for all $z \neq x_{n-1}$ such that $x<z \prec y$. A poset is said to be strongly lexicographically shellable (or SL-shell$a b l e)$ if it is graded and admits an $S L$-labeling.

Figure $1 \mathrm{~b}$ above (Example 2.4) shows an $S L$-labeling, while Figure la shows an $L$-labeling which is not $S L$. 
Proposition 3.5. If $P$ is an $S L$-shellable poset, then its order dual $P^{*}$ is also SL-shellable.

Proof. If $\lambda: C(P) \rightarrow \Lambda$ is an $S L$-labeling of $P$, then $\lambda^{*}\left(x<{ }^{*} y\right)=\lambda(y \prec x)$ defines an $S L$-labeling $\lambda^{*}: C\left(P^{*}\right) \rightarrow \Lambda^{*}$ of $P^{*}$.

Proposition 3.6. Let $L$ be a graded lattice with join-irreducibles $I(L)$. Then conditions (i) and (ii) below are equivalent and both imply (iii):

(i) there is an injective admissible map $\omega: I(L) \rightarrow \mathbf{P}$,

(ii) there is an admissible map $\omega: I(L) \rightarrow \mathbf{P}$ such that under the induced edge-labeling $\gamma$ of $L$ all rising chains $x_{0} \prec x_{1} \prec \cdots \prec x_{n}$ have strictly increasing JordanHölder sequences $\gamma\left(x_{0}, x_{1}\right)<\gamma\left(x_{1}, x_{2}\right)<\cdots<\gamma\left(x_{n-1}, x_{n}\right)$.

(iii) $L$ is $S L$-shellable.

Proof. (i) implies (ii): The same map $\omega$ will do.

(ii) implies (i): The map $\omega: I(L) \rightarrow \mathrm{P}$ induces a linearly ordered partition of $I(L)$ with blocks $\omega^{-1}(n), n \in$ range $\omega$. Let $\omega^{\prime}: I(L) \rightarrow \mathbf{P}$ be an injective map such that $\omega^{\prime}\left(t_{1}\right)<\omega^{\prime}\left(t_{2}\right)$ if $\omega\left(t_{1}\right)<\omega\left(t_{2}\right), t_{1}, t_{2} \in I(L)$. Such a map $\omega^{\prime}$ clearly exists. If $x \prec z \prec y$ in $L$ then, by assumption either $\gamma(x, z)<\gamma(z, y)$ or $\gamma(x, z)>\gamma(z, y)$. It is easy to check that $\gamma(x, z)<\gamma(z, y)$ if and only if $\gamma^{\prime}(x, z)<\gamma^{\prime}(z, y)$, where $\gamma^{\prime}$ is the edge-labeling of $L$ which $\omega^{\prime}$ induces. Since $\gamma$ is an $R$-labeling, we must conclude that $\gamma^{\prime}$ is also an $R$-labeling. Hence, $\omega^{\prime}$ is admissible.

(ii) implies (iii): It is already known that the induced edge-labeling $\gamma$ of $L$ is an $L$-labeling (Theorem 3.1), and we shall verify that under condition (ii) it is an $S L$-labeling by induction on the length of intervals of $L$. If $[x, y]$ is an interval of length one there is nothing to prove. Assume that $\gamma$ is an $S L$-labeling of all intervals of length $\langle n-1, n \geqslant 2$, and let $[x, y]$ be an interval of length $n$ with unique rising chain $x=x_{0} \prec x_{1} \prec \cdots \prec x_{n}=y$. Suppose that $z \neq x_{n-1}$ and $x<z \prec y$. Also, let $t \in I(L)$ such that $x<t \vee x=x_{1}$ and $\gamma\left(x, x_{1}\right)=\omega(t)$. If $t<z$, then $x_{1}=t \vee x<z$, and since $\left[x_{1}, y\right]$ is an interval of length $n-1$ and $x_{1} \prec x_{2} \prec \cdots \prec x_{n}=y$ must be its unique rising chain, the induction assumption forces $\gamma(z, y)<\gamma\left(x_{n-1}, y\right)$. If $t \$ z$, then $\gamma(z, y)<\omega(t)$ since $t<y$. Hence, $\gamma(z, y)<\omega(t)=\gamma\left(x, x_{1}\right)<\gamma\left(x_{n-1}, y\right)$, where the final inequality is forced by condition (ii).

The implication (ii) $\Rightarrow$ (iii) of the preceding proposition can be made more precise: Let $L$ be a graded lattice, $\omega: I(L) \rightarrow \mathbf{P}$ an admissible map and $\gamma$ the induced edge-labeling of $L$. Then $\gamma$ is an $S L$-labeling if and only if whenever $x_{0} \prec x_{1} \prec \cdots \prec x_{n}$ and $\gamma\left(x_{0}, x_{1}\right)=\gamma\left(x_{1}, x_{2}\right)=\cdots=\gamma\left(x_{n-1}, x_{n}\right)$ then $\left[x_{0}, x_{n}\right]$ is a chain. We omit the proof.

The induced edge-labeling of an admissible map does not have to be an SL-labeling as the following counterexample shows (Figure 2).

We do not know whether all admissible lattices are $S L$-shellable, but we can show that the important examples are. 


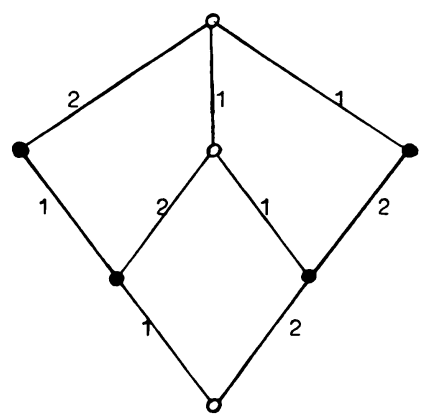

FIGURE 2

THEOREM 3.7. Finite upper-semimodular, lower-semimodular and supersolvable lattices are $S L$-shellable.

Proof. R. Stanley has shown that for every finite supersolvable lattice $L$ there is an admissible map $\omega$ which satisfies condition (ii) of Proposition 3.6 [19, Proposition 2.4 , p. 363]. He has also proved that any injective order-preserving map $\omega$ : $I(L) \rightarrow P$ is admissible when $L$ is finite and upper-semimodular [19, Proposition 2.2, p. 362]. Hence, Propositions 3.5 and 3.6 imply the present result.

REMARK. Extending Stanley's above-mentioned result I. Rival has shown [16, Theorem 2, p. 102] that a finite lattice $L$ is upper-semimodular if and only if every injective order-preserving map $\omega: I(L) \rightarrow P$ is admissible. As the following example of a modular lattice shows (Figure 3), an admissible map does not have to be order-preserving. Hence, Proposition 2.1 in $[19$, p. 362] is false.

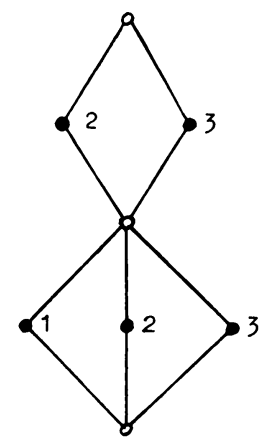

FIGURE 3

EXAMPLE 3.8. Let $G$ be a finite geometric lattice. K. Baclawski has observed [23, Corollary 4.3] that $G-\{x\}$ is a shellable poset for every $x \in G$. We will show that in fact all chains and certain antichains can be removed from $G$ without losing shellability. The case of chains has independently been considered and further generalized by Baclawski [24].

Suppose that c: $x_{1}<x_{2}<\cdots<x_{k}$ is a chain in $\bar{G}$. Let $I(G)$ be the set of join-irreducibles (atoms) of $G$, and let $\omega: I(G) \rightarrow[n]$ be a bijection such that $p<x_{i}<q \vee x_{i}$ implies $\omega(q)<\omega(p)$ for all $p, q \in I(G)$ and $x_{i} \in$ c. Since $G$ is upper-semimodular we know that the map $\omega$ is admissible and hence induces an 
$L$-labeling $\gamma$ of $G$ in the usual way. It is easy to verify that if $y \prec x_{i} \prec z$ in $G$ with $x_{i} \in \mathrm{c}$ then $\gamma\left(y, x_{i}\right)>\gamma\left(x_{i}, z\right)$. Hence, by Proposition 2.8 the subposet $G-\mathrm{c}$ is $L$-shellable under the restriction of $\gamma$. Now, a maximal chain of $G$ with strictly decreasing Jordan-Hölder sequence either is contained in $G-\mathbf{c}$ or includes an element of c. Counting the chains of the latter type by the principle of inclusion-exclusion and using Theorem 2.7 we are led to the following formula:

$$
\begin{array}{r}
\mu(G-\mathbf{c})=\mu(G)+\sum_{e=1}^{k}(-1)^{e} \sum_{1<i_{1}<i_{2}<\cdots<i_{e}<k} \mu\left(\hat{0}, x_{i_{1}}\right) \mu\left(x_{i_{1}}, x_{i_{2}}\right) \\
\cdots \mu\left(x_{i_{e-1}}, x_{i_{e}}\right) \mu\left(x_{i_{e}}, \hat{1}\right) .
\end{array}
$$

Also certain antichains can be deleted from geometric lattices without losing shellability. Apart from the case of full rank levels (cf. Theorem 4.1 below) we have the following. Let $G$ be a finite geometric lattice, $S=\left\{x_{1}, x_{2}, \ldots, x_{s}\right\}$ a subset of $\bar{G}$, and let $A$ be the set of atoms under $S$, that is, $A=\{p \in I(G) \mid p<x$ for some $x \in S$ \}. Assume that $S$ has the property that if $x<z$ in $G$ and $x \in S$ then $a<z$ for some atom $a \in I(G)-A$. In particular, $S$ must be an antichain. Now, choose a bijection $\omega: I(G) \rightarrow[n]$ such that $\omega(I(G)-A)=[n-|A|]$. As before, it is easy to check that the induced $L$-labeling $\gamma$ of $G$ has the property that $\gamma(y, x)>\gamma(x, z)$ when $y \prec x \prec z$ in $G$ and $x \in S$. So again by Proposition 2.8 we find that the subposet $G-S$ is $L$-shellable. Counting maximal chains with strictly decreasing Jordan-Hölder sequences in $G$ and $G-S$ we obtain the formula:

$$
\mu(G-S)=\mu(G)-\sum_{i=1}^{s} \mu\left(\hat{0}, x_{i}\right) \mu\left(x_{i}, \hat{1}\right) .
$$

Examples of antichains $S$ which satisfy the above requirement include the set of maximal complements of a fixed element in $G$. In particular, if $S$ is the set of all complements of a modular element in $G$, then (3.10) in combination with $\mathbf{H}$. Crapo's complementation theorem shows that $\mu(G-S)=0$, so $G-S$ is acyclic.

4. Constructing shellable posets. This section is devoted to showing a number of ways in which shellable posets can be constructed from other shellable or $L$-shellable posets.

Let $P$ be a finite pure poset of length $r-1$. Then $\hat{P}$ is graded with rank function $\rho$, and $\rho(x) \in[r]$ for all $x \in P$. For any subset $S \subseteq[r]$ we define $P_{S}=\{x \in$ $P \mid \rho(x) \in S\}$. This definition is slightly more general than the definition of rankselected subposets given in $\$ 2$, where only bounded posets were considered.

THEOREM 4.1. If $P$ is a shellable poset of length $r-1$, then $P_{S}$ is shellable for all $S \subseteq[r]$.

Proof. Fix a subset $S \subseteq[r]$ and let $\mathscr{N}$ and $\Re_{s}$ denote the sets of maximal chains of $P$ and $P_{S}$ respectively. Assume that a certain linear order $\Omega$ of the set $\mathfrak{R}$ is a shelling. For every $c \in \mathfrak{R}_{s}$ define $\theta(c)$ to be the least element under $\Omega$ of the set $\{\mathbf{m} \in \mathfrak{K} \mid \mathbf{c} \subseteq \mathbf{m}\}$. This determines an injective map $\theta: \mathfrak{R}_{s} \rightarrow \mathfrak{R}$, and we derive a linear order $\Omega^{\prime}$ of the set $\mathfrak{N}_{s}$ by the rule: $c<\Omega^{\prime} \mathbf{d}$ if and only if 


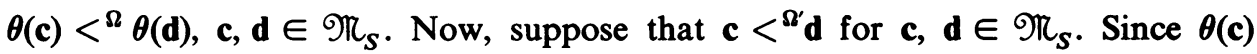
$<^{\Omega} \theta(d)$ and $\Omega$ is a shelling, we know that there exists a maximal chain $\mathbf{m} \in \Re$ such that $\mathbf{m}<^{\Omega} \theta(d)$ and

$$
\theta(c) \cap \theta(d) \subseteq \mathbf{m} \cap \theta(d)=\theta(d)-\{x\} \text { for some } x \in \theta(d) .
$$

In case $x \notin \mathbf{d}$, then $\mathbf{d} \subseteq \theta(\mathbf{d})-\{x\} \subseteq \mathbf{m}$, which contradicts the definition of $\boldsymbol{\theta}$ (d) since $\mathbf{m}<^{\Omega} \theta(d)$. Hence $x \in d$. Let $b=\mathbf{m} \cap P_{s}$. Then $b \in \Re_{s}$ and $\theta(b) \leqslant \mathbf{m}<$ $\theta(d)$ so $b<\Omega^{\Omega^{\prime}}$ d. Applying the operator $P_{S} \cap \cdot$ to the formula (*) we get $\mathbf{c} \cap \mathbf{d} \subseteq \mathrm{b}$ $\cap \mathrm{d}=\mathrm{d}-\{x\}$. Hence, $\Omega^{\prime}$ is a shelling of the set $\Re_{s}$.

Proposition 4.2. If $P$ is an ( $L-)$ shellable poset then all intervals of $P$ are $(L-)$ shellable.

Proof. Suppose that $P$ is shellable and that $[x, y]$ is an interval of $P$. Let c: $x_{1} \prec x_{2} \prec \cdots \prec x_{g}=x$ and $\mathrm{d}: y=y_{1} \prec y_{2} \prec \cdots \prec y_{h}$ be two unrefinable chains in $P$ such that $x_{1}$ is a minimal and $y_{h}$ is a maximal element. Let $\mathbf{m}_{1}, \mathbf{m}_{2}, \ldots, \mathbf{m}_{t}$ be the maximal chains in $P$ which contain $\mathbf{c} \cup \mathbf{d}$, and assume that they are listed in the order in which they appear in the shelling of $P$. It is straightforward to verify that $\left(\left(\mathbf{m}_{i}-(\mathbf{c} \cup \mathbf{d})\right) \cup\{x, y\}\right)_{i=1}^{t}$ is a shelling of $[x, y]$.

In case $P$ is $L$-shellable the conclusion is immediate from Definition 2.1.

Let $P$ and $Q$ be two posets. The direct product $P \times Q$ is the poset defined on the product set by $(x, y) \leqslant\left(x^{\prime}, y^{\prime}\right)$ if and only if $x<x^{\prime}$ in $P$ and $y<y^{\prime}$ in $Q$. The ordinal sum $P \oplus Q$ is the poset on the disjoint union of $P$ and $Q$ defined by the rule: $x<y$ in $P \oplus Q$ if and only if (i) $x, y \in P$ and $x<y$ in $P$, or (ii) $x, y \in Q$ and $x<y$ in $Q$, or (iii) $x \in P$ and $y \in Q$.

Theorem 4.3. $P \times Q$ is L-shellable if and only if both $P$ and $Q$ are L-shellable.

Proof. $P \times Q$ is clearly a graded poset if and only if both $P$ and $Q$ are. Let $\lambda$ : $C(P) \rightarrow \Lambda$ and $\lambda^{\prime}: C(Q) \rightarrow \Lambda^{\prime}$ be $L$-labelings of the graded posets $P$ and $Q$. A covering relation $(x, y) \prec\left(x^{\prime}, y^{\prime}\right)$ occurs in $P \times Q$ if and only if $x=x^{\prime}$ and $y<y^{\prime}$ or $x \prec x^{\prime}$ and $y=y^{\prime}$. Define an edge-labeling $\lambda^{\prime \prime}: C(P \times Q) \rightarrow \Lambda \oplus \Lambda^{\prime}$ by $\lambda^{\prime \prime}\left((x, y) \prec\left(x^{\prime}, y^{\prime}\right)\right)=\lambda\left(x \prec x^{\prime}\right)$ if $y=y^{\prime}$ and $\lambda^{\prime \prime}\left((x, y) \prec\left(x^{\prime}, y^{\prime}\right)\right)=\lambda^{\prime}\left(y \prec y^{\prime}\right)$ if $x=x^{\prime}$. It is straightforward to verify that $\lambda^{\prime \prime}$ is an $L$-labeling of $P \times Q$.

If $P \times Q$ is $L$-shellable then, by Proposition 4.2 , so are $P \simeq[(\hat{0}, \hat{0}),(\hat{1}, \hat{0})]$ and $Q \simeq[(\hat{0}, \hat{0}),(\hat{0}, \hat{1})]$.

THEOREM 4.4. Let $P$ and $Q$ be two posets such that $\hat{P}$ and $\hat{Q}$ are L-shellable. Then $\hat{X}$ is L-shellable, where $X=P \oplus Q$. Also, $P \oplus Q$ is shellable if and only if both $P$ and $Q$ are.

Proof. Suppose that $\lambda: C(\hat{P}) \rightarrow \Lambda$ and $\lambda^{\prime}: C(\hat{Q}) \rightarrow \Lambda^{\prime}$ are $L$-labelings of the graded posets $\hat{P}$ and $\hat{Q}$. Define an edge-labeling $\lambda^{\prime \prime}: C(\hat{X}) \rightarrow\left(\Lambda \oplus \Lambda^{\prime}\right) \times\left(\Lambda \oplus \Lambda^{\prime}\right)$ by (i) $\lambda^{\prime \prime}(x \prec y)=(\lambda(x \prec y), \lambda(x \prec y))$ if $x \prec y$ and $y \neq \hat{1}$ in $\hat{P}$, (ii) $\lambda^{\prime \prime}(x \prec y)=$ $\left(\lambda^{\prime}(x \prec y), \lambda^{\prime}(x \prec y)\right)$ if $x \prec y$ and $x \neq \hat{0}$ in $\hat{Q}$, and (iii) $\lambda^{\prime \prime}(x \prec y)=(\lambda(x \prec$ $\left.\hat{1}), \lambda^{\prime}(\hat{0} \prec y)\right)$ if $x<\hat{1}$ in $\hat{P}$ and $\hat{0} \prec y$ in $\hat{Q}$. $\lambda^{\prime \prime}$ is an $L$-labeling of $\hat{X}$. We omit the easy verification. 
Suppose that $\mathbf{m}_{1}, \mathbf{m}_{2}, \ldots, \mathbf{m}_{s}$ is a shelling of $P$ and that $\mathbf{m}_{1}^{\prime}, \mathbf{m}_{2}^{\prime}, \ldots, \mathbf{m}_{t}^{\prime}$ is a shelling of $Q$. Then $\left\{\mathbf{c}_{i j}\right\}_{1<i<s, 1<j<t}$, where $\mathbf{c}_{i j}=\mathbf{m}_{i} \cup \mathbf{m}_{j}^{\prime}$, is the set of maximal chains of $P \oplus Q$, and it is easy to check that the lexicographic order of the indices determines a shelling of $P \oplus Q$. If on the other hand $P \oplus Q$ is shellable then so are both $P$ and $Q$ by Theorem 4.1 .

The cardinal power $Q^{P}$ of two posets $P$ and $Q$ is the set of order-preserving maps $f: P \rightarrow Q$ partially ordered by $f<g$ if and only if $f(x)<g(x)$ for all $x \in P$.

Theorem 4.5. Let $P$ and $Q$ be two finite posets. Then $Q^{P}$ is $L$-shellable if and only if $Q$ is $L$-shellable.

Proof. First note that $Q^{P}$ is bounded if and only if $Q$ is bounded. Also, if $Q$ is bounded then $Q \simeq[f, g]$ in $Q^{P}$, where $f(x)=\hat{0}$ for all $x \in P$ and $g(x)=\hat{0}$ for all $x \in P$ with the exception of a maximal element $m \in P$ for which $g(m)=\hat{1}$. Hence, by Proposition 4.2 the "only if" part is done.

Let $\lambda: C(Q) \rightarrow \Lambda$ be an $L$-labeling of the graded poset $Q$. It is easy to see that $f<g$ in $Q^{P}$ if and only if $f(x)=g(x)$ for all $x \in P$ with one exception $p \in P$ for which $f(p) \prec g(p)$. Hence, in particular, $Q^{P}$ is also graded. Now, let $\sigma: P \rightarrow Z$ be an order-reversing injective map from $P$ to the integers. Define an edge-labeling $\lambda^{\prime}$ : $C\left(Q^{P}\right) \rightarrow \mathbf{Z} \otimes \Lambda$ by $\lambda^{\prime}(f \prec g)=(\sigma(p), \lambda(f(p) \prec g(p)))$ where $p$ is the unique element of $P$ such that $f(p) \prec g(p)$. Here $\mathbf{Z} \otimes \Lambda$ denotes the ordinal product, which is defined by the lexicographic order on the product set: $(n, \lambda)<\left(n^{\prime}, \lambda^{\prime}\right)$ if and only if $n<n^{\prime}$ or $n=n^{\prime}$ and $\lambda \leqslant \lambda^{\prime}$. Again, we leave the verification that $\lambda^{\prime}$ is an $L$-labeling to the reader.

The preceding result reconfirms that finite distributive lattices are $L$-shellable, since by a theorem of G. Birkhoff [2, Theorem 3, p. 59] $L \simeq 2^{I(L)}$, where $L$ is a finite distributive lattice and $I(L)$ its subposet of join-irreducibles.

The interval poset $\operatorname{Int}(P)$ of a poset $P$ is the set of all intervals of $P$ ordered by containment. Thus $[x, y]<[u, v]$ in $\operatorname{Int}(P)$ if and only if $u<x<y<v$ in $P$. By convention we also adjoin the empty interval $\varnothing$ to $\operatorname{Int}(P)$, so that $\operatorname{Int}(P)$ is bounded if and only if $P$ is bounded.

Theorem 4.6. Int $(P)$ is $L$-shellable if and only if both $P$ and its order dual $P^{*}$ are L-shellable.

Proof. Suppose that $P$ is a graded poset and that $\lambda: C(P) \rightarrow \Lambda$ and $\lambda^{\prime}$ : $C\left(P^{*}\right) \rightarrow \Lambda^{\prime}$ are $L$-labelings. Define an edge-labeling $\lambda^{\prime \prime}: C(\operatorname{Int}(P)) \rightarrow \Lambda^{\prime} \oplus \mathbf{Z} \oplus \Lambda$ by (i) $\lambda^{\prime \prime}([x, y] \prec[u, v])=\lambda(y \prec v)$ if $x=u$, (ii) $\lambda^{\prime \prime}([x, y] \prec[u, v])=\lambda^{\prime}\left(x \prec{ }^{*} u\right)$ if $y=v$, and (iii) $\lambda^{\prime \prime}(\varnothing \prec[x, x])=\rho(x)$, where $\rho$ denotes the rank function in $P$. It is not hard to verify that $\lambda^{\prime \prime}$ is an $L$-labeling of $\operatorname{Int}(P)$.

If $P$ is a bounded poset then clearly $P \simeq[[\hat{0}, \hat{0}],[\hat{0}, \hat{1}]]$ and $P^{*} \simeq[[\hat{1}, \hat{1}],[\hat{0}, \hat{1}]]$ in $\operatorname{Int}(P)$. Hence, if $\operatorname{Int}(P)$ is $L$-shellable then by Proposition 4.2 so are $P$ and $P^{*}$.

The preceding Theorems $4.2-4.6$ remain true if " $L$-shellable" is everywhere replaced by " $S L$-shellable" (cf. Definition 3.4). In particular $\operatorname{Int}(P)$ is $S L$-shellable if and only if $P$ is $S L$-shellable. 
5. Face-lattices of complexes. If we order the faces of a simplicial or polyhedral complex $K$ by inclusion, we get a poset $P_{K}$. Adjoining a greatest element $\hat{1}$ to $P_{K}$ we obtain a lattice $L_{K}$, the face-lattice of $K$. The order complex $\Delta\left(\bar{L}_{K}\right)$ is known in topology as the barycentric subdivision sd $K$ of $K$. We shall in this section discuss some cases for which the shellability of $K$ is inherited by $P_{K}$ (or, equivalently, $L_{K}$ or $\left.\bar{L}_{K}\right)$ :

THEOREM 5.1. Let $K$ be a shellable simplicial complex. Then the barycentric subdivision sd $K$ is shellable.

Proof. Suppose that $f_{1}, f_{2}, \ldots, f_{t}$ are the facets of $K$ arranged in a shelling order. The poset $P_{K}$ of faces of $K$ is a pure poset of length $=n=\operatorname{dim} K+1$, with maximal elements $f_{1} f_{2}, \ldots, f_{t}$ and least element $\hat{0}=\varnothing$. Since sd $K=\Delta\left(P_{K}-\{\hat{0}\}\right)$ the theorem will follow if we construct a shelling order $\Omega$ of the set $\mathfrak{N}$ of maximal chains of $\boldsymbol{P}_{\boldsymbol{K}}$.

Let $\mathfrak{N}_{i}$ be the set of all maximal chains of $P_{K}$ which contain $f_{i}$, so that $\mathfrak{N}=\bigcup_{i=1}^{t} \mathfrak{M}_{i}$. For every $i, i=1,2, \ldots, t,\left[\hat{0}, f_{i}\right]$ is a finite Boolean algebra on $n$ atoms. Suppose that the elements which are covered by $f_{i}$ are labeled $c_{i 1}, c_{i 2}, \ldots, c_{i n}$ in such a way that for $j=1,2, \ldots, k_{i}$ the element $c_{i j}$ is also covered by $f_{e}$ for some $e<i$, but for $j=k_{i}+1, k_{i}+2, \ldots, n$ this is not the case. Since $K$ is shellable $k_{i} \geqslant 1$ when $i>1$. The map $\omega: c_{i j} \mapsto j$ is an admissible map from the join-irreducibles of the dual lattice $\left[\hat{0}, f_{i}\right]^{*}$, and therefore it induces an $L$-labeling, which in turn lexicographically generates a shelling order of $\mathfrak{T l}_{i}$ (cf. Theorems 2.3 and 3.1). Let the elements of $\Re_{i}$ be called $\mathbf{m}_{i 1}, \mathbf{m}_{i 2}, \ldots, \mathbf{m}_{i n !}$, where the second index is compatible with the shelling order we have obtained. The important feature of this order, except that it shells $\mathfrak{T l}_{i}$, is that whenever $c_{i e_{1}} \in \mathbf{m}_{i j_{1}}$, $c_{i e_{2}} \in \mathbf{m}_{i j_{2}}$ and $e_{1} \leqslant k_{i}<e_{2}$, then $j_{1}<j_{2}$. Suppose the above process has been carried out for each $i, 1 \leqslant i \leqslant t$, so that $\mathscr{N}=\left\{\mathbf{m}_{i j}\right\}_{1<i<t, 1<j<n !}$. The lexicographic order of the indices defines a linear order $\Omega$ on $\Re$.

To verify that $\Omega$ is a shelling, assume given $\mathbf{m}_{i_{0} j_{0}} \in \mathscr{N}$. If $\mathbf{m}_{i_{1} j_{1}}<\boldsymbol{\Omega} \mathbf{m}_{i_{0} j_{0}}$ then either (a) $i_{1}=i_{0}$ and $j_{1}<j_{0}$ or (b) $i_{1}<i_{0}$. In case (a), $\mathbf{m}_{i_{1} j_{1}}$ and $\mathbf{m}_{i_{0} j_{0}}$ both belong to $\mathfrak{N}_{i_{0}}$ and since the second index determines a shelling of $\mathfrak{T}_{i_{0}}$ we may conclude the existence of a $\mathbf{m}_{i_{0} j_{2}} \in \Re_{i_{0}}$ with $j_{2}<j_{0}$ and $\mathbf{m}_{i_{1} j_{1}} \cap \mathbf{m}_{i_{0_{j}}} \subseteq \mathbf{m}_{i_{0} j_{2}} \cap \mathbf{m}_{i_{0} j_{0}}=\mathbf{m}_{i_{0} j_{0}}$ $\{x\}$ for some $x \in \mathbf{m}_{i_{0} j_{0}}$. Case (b) must be further subdivided into two subcases as follows. Suppose that $\mathbf{m}_{i_{0} j_{0}}: \hat{0}=x_{0} \prec x_{1} \prec \cdots \prec x_{n}=f_{i_{0}}$ and that $x_{n-1}=c_{i_{0}}$. In case $e<k_{i_{0}}$ then $x_{n-1} \prec f_{i_{2}}$ for some $i_{2}<i_{0}$, so $\mathrm{m}_{i_{2} j_{2}}: x_{0} \prec x_{1} \prec \cdots \prec x_{n-1} \prec f_{i_{2}}$, which precedes $\mathbf{m}_{i_{0} j_{0}}$ under $\Omega$, satisfies $\mathbf{m}_{i_{1} j_{1}} \cap \mathbf{m}_{i_{0} j_{0}} \subseteq \mathbf{m}_{i_{2} j_{2}} \cap \mathbf{m}_{i_{0} j_{0}}=\mathbf{m}_{i_{0} j_{0}}-\left\{f_{i_{0}}\right\}$. Assume next that $e>k_{i_{0}}$. Let $g=\max \left\{h \mid x_{h} \in \mathbf{m}_{i_{1} j_{1}} \cap \mathbf{m}_{i_{0} j_{0}}\right\}$. Then $0<g<n-2$. Since $f_{1}, f_{2}, \ldots, f_{t}$ is a shelling of $K$ we know that $x_{g}<c_{i_{0} d}<f_{i_{0}}$ for some $d<k_{i_{0}}$. Let $x_{g}=y_{0} \prec y_{1} \prec \cdots \prec y_{n-g-1}=c_{i_{0} d}$ be an arbitrary unrefinable chain from $x_{g}$ to $c_{i_{0 d} d}$. Then $\mathbf{m}_{i_{0} j_{2}}: x_{0} \prec x_{1} \prec \cdots \prec x_{g}=y_{0} \prec y_{1} \prec \cdots \prec y_{n-g-1}=c_{i_{\alpha d}} \prec$ $f_{i_{0}}$ precedes $\mathbf{m}_{i_{0} j_{0}}$ in the shelling order of $\mathfrak{R}_{i_{0}}$. Hence, there exists a $\mathbf{m}_{i_{0} j_{3}} \in \mathfrak{T}_{i_{0}}$ with $j_{3}<j_{0}$ such that $\mathbf{m}_{i_{0} j_{2}} \cap \mathbf{m}_{i_{0} j_{0}} \subseteq \mathbf{m}_{i_{\alpha_{3}}} \cap \mathbf{m}_{i_{0} j_{0}}=\mathbf{m}_{i_{0_{j} j_{0}}}-\{x\}$ for some $x \in \mathbf{m}_{i_{0} j_{0}}$. Since $\mathbf{m}_{i_{1} j_{1}} \cap \mathbf{m}_{i_{a_{0}}} \subseteq\left\{x_{0}, x_{1}, \ldots, x_{g}\right\} \subseteq \mathbf{m}_{i_{0} j_{2}} \cap \mathbf{m}_{i_{0} j_{0}}$, this concludes the proof. 
It has been proved by Bruggesser and Mani that the boundary complex of a convex polytope is shellable [3, p. 202]. Their result together with the preceding theorem shows that the face-lattice of a simplicial convex polytope is shellable. However, more is true. As seems to have been first observed by Ewald and Shephard [5, p. 10] the barycentric subdivision of the boundary complex of a convex polytope is isomorphic to the boundary complex of some simplicial convex polytope. This fact together with Bruggesser and Mani's result shows the following.

Proposition 5.2. The face-lattice of a convex polytope is shellable.

It is possible to modify our proof above of Theorem 5.1, by introducing induction on length, so that in combination with Bruggesser and Mani's argument [3, pp. 202-3] it yields Proposition 5.2. We leave the details aside. A similar proof has independently been found by S. Provan [13].

In this connection we would like to raise the question: whether the face-lattices of convex polytopes are $L$-shellable? The face-lattice of any simplex is of course $S L$-shellable, since such a lattice is Boolean. An $S L$-labeling of the face-lattice of a 2-dimensional polytope is shown in Figure 4.

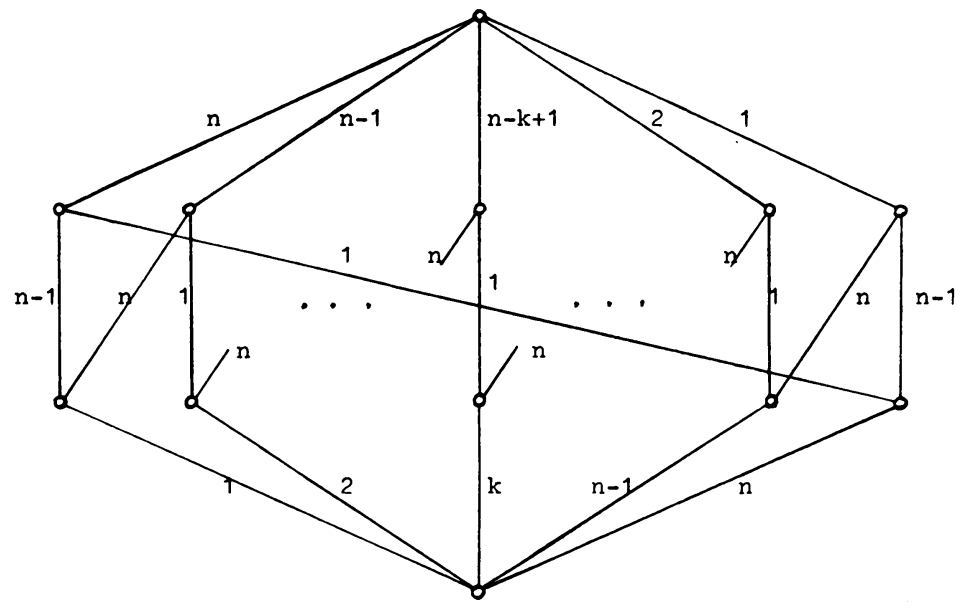

FIGURE 4

Let $L(P)$ denote the face-lattice of a convex polytope $P$. B. Lindström has asked [11] whether it is true for every convex polytope $P$ that there exists a convex polytope $Q$ such that the interval lattice $\operatorname{Int}(L(P))$ is isomorphic to $L(Q)$. Lindström observed that if $P$ is the $n$-dimensional simplex, then $Q$ is the $(n+1)$ dimensional cube. It is easy to see also that if $P$ is 2 -dimensional then $Q$ is the dual of a 3-dimensional antiprism. Hence, by Theorem 4.6, the face-lattices of cubes of all dimensions and their duals and also of 3-dimensional antiprisms and their duals are $S L$-shellable.

6. Locally semimodular posets. A finite poset $P$ is said to be semimodular if whenever two distinct elements $u, v \in P$ both cover $t \in P$ there is a $z \in P$ which covers each of $u$ and $v . P$ is said to be locally semimodular when all intervals $[x, y]$ 
of $P$ are semimodular. As was apparently first noticed by $O$. Ore, bounded semimodular finite posets are pure. However, such posets need not be CohenMacaulay. It was shown independently by K. Baclawski [1] and F. Farmer [0] that bounded locally semimodular finite posets are Cohen-Macaulay over $\mathbf{Z}$. Notice that a lattice is locally semimodular as a poset if and only if it is upper-semimodular in the usual sense.

THEOREM 6.1. Suppose that a finite poset $P$ is bounded and locally semimodular. Then $P$ is shellable.

Proof. Let us say that a linear order $\Omega$ of the set of maximal chains $\mathscr{N}$ of a graded poset $Q$ has property $\Psi$ if whenever $\mathbf{m}, \mathbf{m}^{\prime} \in \mathfrak{N}, \mathbf{m}^{\prime}: \hat{0}=y_{0} \prec y_{1} \prec \cdots \prec$ $y_{n}=\hat{1}, \mathbf{m}: \hat{0}=x_{0} \prec x_{1} \prec \cdots \prec x_{n}=\hat{1}, x_{i}=y_{i}$ for $i=0,1, \ldots, e$ and $x_{e+1} \neq$ $y_{e+1}$, then

$(\alpha)$ if $\left\{y_{0}, y_{1}, \ldots, y_{e+1}\right\} \subseteq \mathbf{m}^{\prime \prime} \in \mathscr{N}$ and $\mathbf{m}^{\prime}<\mathbf{\Omega}$ m then $\mathbf{m}^{\prime \prime}<\mathbf{s} \mathbf{m}$, and

$(\beta)$ if $\mathbf{m}^{\prime}-\left\{y_{e}\right\} \subseteq \mathbf{m}^{\prime \prime}$ for some $\mathbf{m}^{\prime \prime} \in \mathscr{T}$ such that $\mathbf{m}^{\prime \prime}<^{\mathbf{\Omega}} \mathbf{m}$ but $\mathbf{m}-\left\{x_{e}\right\} \mathbb{Z}$ $\mathbf{m}^{\prime \prime \prime}$ for all $\mathbf{m}^{\prime \prime \prime} \in \mathcal{N}$ such that $\mathbf{m}^{\prime \prime \prime}<^{\Omega} \mathbf{m}$ then $\mathbf{m}^{\prime}<^{\Omega} \mathbf{m}$.

We shall prove for every graded poset that the set of maximal chains can be given a linear order having property $\Psi$. Since for posets of length 2 every linear order trivially posesses property $\Psi$, we may proceed by induction on length.

Suppose that $Q$ is a graded poset of length $n, n>3$, and let $Q^{\prime}$ denote the rank-selected subposet $Q_{[n-2]}=\{x \in Q \mid \rho(x) \neq n-1\}$. $Q^{\prime}$ is graded and of length $n-1$, so by the induction assumption there is a linear order $\Omega^{\prime}$ with property $\Psi$ of the set $\Re^{\prime}$ of maximal chains of $Q^{\prime}$. Label the elements of $\Re^{\prime}, \mathbf{m}_{1}^{\prime}, \mathbf{m}_{2}^{\prime}, \ldots, \mathbf{m}_{s}^{\prime}$, in such a way that the natural order of the indices expresses the order $\Omega^{\prime}$. For $\mathbf{m}_{i}^{\prime} \in \mathfrak{N}^{\prime}, \mathbf{m}_{i}^{\prime}: \hat{0}=x_{0} \prec x_{1} \prec \cdots \prec x_{n-2}<\hat{1}$, let $A_{i}=\left\{z \in Q \mid x_{n-2} \prec z \prec \hat{1}\right\}$, $B_{i}=\left\{z \in A_{i} \mid\right.$ there is a $y \in Q$ such that $x_{n-3} \prec y \prec z$ and $\left(\mathbf{m}_{i}^{\prime}-\left\{x_{n-2}\right\}\right) \cup\{y\}$ $\left.<^{\Omega^{\prime}} \mathbf{m}_{i}^{\prime}\right\}$, and $C_{i}=A_{i}-B_{i}$. Label the elements of $A_{i}, z_{i 1}, z_{i 2}, \ldots, z_{i a_{1}}, a_{i}=\left|A_{i}\right|$, in such a way that if $z_{i j} \in B_{i}$ and $z_{i k} \in C_{i}$ then $j<k$. Let $\mathbf{m}_{i j}=\mathbf{m}_{i}^{\prime} \cup\left\{z_{i j}\right\}$, for $i=1,2, \ldots, s$ and $j=1,2, \ldots, a_{i}$. Then the lexicographic order of the indices determines a linear order $\Omega$ of the set $\mathscr{R}=\left\{\mathbf{m}_{i j} \mid 1<i<s, 1<j<a_{i}\right\}$ of maximal chains of $Q$. We claim that $\Omega$ has property $\Psi$.

To validate this claim, let us suppose that $\mathbf{m}, \mathbf{m}^{\prime} \in \mathfrak{R}, \mathbf{m}^{\prime}: \hat{0}=y_{0} \prec y_{1}$ $\prec \cdots \prec y_{n}=\hat{1}, \mathbf{m}: \hat{0}=x_{0} \prec x_{1} \prec \cdots \prec x_{n}=\hat{1}, x_{i}=y_{i}$ for $i=0,1, \ldots, e$ and $x_{e+1} \neq y_{e+1}$. Then $0 \leqslant e \leqslant n-2$. First consider the case $e=n-2$. Then $\mathbf{m}^{\prime}=\mathbf{m}_{i j}$ and $\mathbf{m}=\mathbf{m}_{i k}$ for some $i, j, k, 1<i<s, 1<j, k<a_{i}$. Condition $(\alpha)$ above is trivially satisfied since $\mathbf{m}^{\prime \prime}$ must equal $\mathbf{m}^{\prime}$. The hypothesis of condition $(\beta)$ is equivalent to $y_{n-1} \in B_{i}$ and $x_{n-1} \in C_{i}$. By construction therefore $j<k$, that is, $\mathbf{m}^{\prime}<\mathbf{\Omega}$. Next, consider the case $0 \leqslant e \leqslant n-3$. Then $\mathbf{m}^{\prime}<^{\Omega} \mathbf{m}$ if and only if $\left(\mathbf{m}^{\prime}-\left\{y_{n-1}\right\}\right)<\Omega^{\prime}\left(\mathbf{m}-\left\{x_{n-1}\right\}\right)$. If $\left\{y_{0}, y_{1}, \ldots, y_{e+1}\right\} \subseteq \mathbf{m}^{\prime \prime} \in \mathscr{R}$, then $\left\{y_{0}, y_{1}, \ldots, y_{e+1}\right\} \subseteq\left(\mathbf{m}^{\prime \prime}-\{z\}\right) \in \mathfrak{T}^{\prime}$ where $z \in \mathbf{m}^{\prime \prime}$ has rank $\rho(z)=n-1$. Hence, since the order $\Omega^{\prime}$ satisfies condition $(\alpha)$, so does $\Omega$. In a similar manner one can verify that property $(\beta)$ is passed along from $\Omega^{\prime}$ to $\Omega$. Consequently, the order $\Omega$ has property $\Psi$, and the induction proof is complete.

Now, suppose that $P$ is a finite poset which is bounded and locally semimodular. 
Then $P$ is pure [2, Theorem 14, p. 40], and hence graded. As we have shown, it is possible to assign a linear order $\Omega$ having property $\Psi$ to the set $\mathscr{N}$ of maximal chains of $P$. It remains to be shown that such an order $\Omega$ is a shelling. Let $\mathbf{m}$, $\mathbf{m}^{\prime} \in \mathfrak{N}, \mathbf{m}: \hat{0}=x_{0} \prec x_{1} \prec \cdots \prec x_{n}=\hat{1}, \mathbf{m}^{\prime}: \hat{0}=y_{0} \prec y_{1} \prec \cdots \prec y_{n}=\hat{1}$, and suppose that $\mathbf{m}^{\prime}<^{\Omega} \mathbf{m}$. Let $d$ be the greatest integer such that $x_{i}=y_{i}$ for $i=0,1, \ldots, d$, and let $g$ be the least integer for which $y_{d+1}<x_{g}$. Since $P$ is locally semimodular, there is an element $z_{d+2}$ which covers both $y_{d+1}$ and $x_{d+1}$ in the interval [ $x_{d}, x_{g}$ ]. Again, if $g>d+2$ there is an element $z_{d+3}$ which covers $z_{d+2}$ and $x_{d+2}$ in $\left[x_{d}, x_{g}\right]$; and so on until the process ends with $z_{g}=x_{g}$ (see Figure 5). Also, let $z_{d+1}=y_{d+1}$.

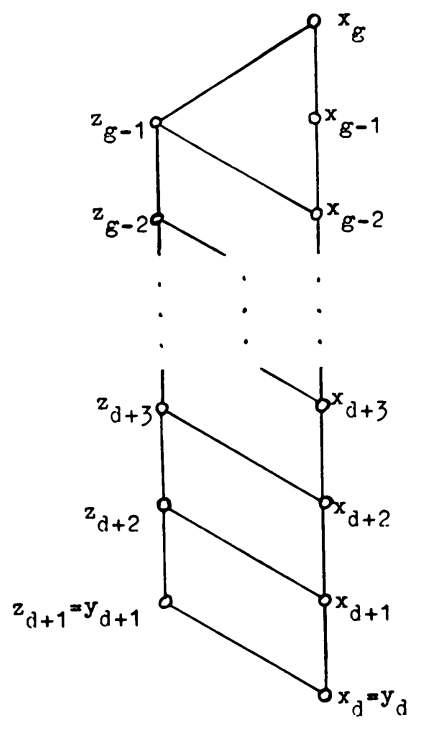

FIGURE 5

Note that, by choice of $g, d+1<e<g-1$ implies $y_{e} \neq x_{e}$ and $z_{e} \neq x_{e}$. For $i=d+1, d+2, \ldots, g-1$ define $\mathbf{m}_{i}$ by $\mathbf{m}_{i}: \hat{0}=x_{0} \prec x_{1} \prec \cdots<x_{i-1} \prec z_{i} \prec$ $z_{i+1} \prec \cdots \prec z_{g-1} \prec x_{g} \prec x_{g+1} \prec \cdots \prec x_{n}=\hat{1}$. We have assumed that $\mathbf{m}^{\prime}$ $<^{\Omega} \mathbf{m}$. Hence, using property $(\alpha)$ above we find that $\mathbf{m}_{d+1}<^{\Omega} \mathbf{m}$. If $\mathbf{m}-\left\{x_{d+1}\right\} \underline{Z}$ $\mathbf{m}^{\prime \prime \prime}$ for all $\mathbf{m}^{\prime \prime \prime} \in \mathscr{N}$ such that $\mathbf{m}^{\prime \prime \prime}<^{\Omega} \mathbf{m}$, then property $(\beta)$ tells us that $\mathbf{m}_{d+2}$ $<^{\Omega} \mathbf{m}$. In that case, if $\mathbf{m}-\left\{x_{d+2}\right\}$ is not included in any maximal chain which precedes $\mathbf{m}$, then $\mathbf{m}_{d+3}<^{\Omega} \mathbf{m}$. Continuing this argument based on property $(\beta)$ we find that either $\mathbf{m}-\left\{x_{e}\right\}$ is contained in an earlier maximal chain for some $e$, $d+1<e<g-2$, or $\mathbf{m}_{g-1}<^{\Omega} \mathbf{m}$. But in the latter case $\mathbf{m}-\left\{x_{g-1}\right\}$ is contained in an earlier maximal chain, namely $\mathbf{m}_{g-1}$. Hence, there is a maximal chain $\mathbf{m}^{\prime \prime} \in \mathfrak{R}$ and an $e, d+1<e<g-1$, such that $\mathbf{m}^{\prime \prime}<^{\mathbf{\Omega}} \mathbf{m}$ and $\mathbf{m}^{\prime} \cap \mathbf{m} \subseteq \mathbf{m}^{\prime \prime} \cap$ $\mathbf{m}=\mathbf{m}-\left\{x_{e}\right\}$. The proof is now complete.

EXAMPLE 6.2. Let $\Pi_{n}$ denote the poset of partitions of the integer $n$ ordered by refinement (see [2, Example 10, p. 16], $\Pi_{6}$ is depicted in Figure $1 \mathrm{~b}$ above). These posets $\Pi_{n}$ as well as their order duals $\Pi_{n}^{*}$ are bounded and semimodular. For $n<7$ 
they are even locally semimodular. This is not true for $n>8$. For instance, the intervals $[(3,2,1,1,1),(5,3)]$ in $\Pi_{8}$ and $[(6,2),(2,2,2,1,1)]$ in $\Pi_{8}^{*}$ are not semimodular. Nevertheless, $\Pi_{8}$ is shellable. For $n>9$ it seems to be unknown even if $\Pi_{n}$ is Cohen-Macaulay.

With any pure simplicial complex $\Delta$ is associated the graph $\Phi_{\Delta}$ defined on the set of all facets of $\Delta$ by letting two facets be adjacent if their intersection is a maximal proper face of each. Let us call $\Delta$ facet-connected if $\Phi_{\Delta}$ is a connected graph. All Cohen-Macaulay complexes are facet-connected. Following Provan [12, p. 25] we shall say that a facet-connected $\delta$-dimensional simplicial complex $\Delta$ with $\nu$ vertices satisfies the Hirsch conjecture if

$$
\operatorname{diam} \Phi_{\Delta}<\nu-\delta-1,
$$

where by diam $\Phi_{\Delta}$ we understand the diameter, that is, the maximal distance between two vertices of $\Phi_{\Delta}$ in the usual graph-theoretic sense. This notion, which stems from polyhedral theory, can be applied to posets by tacitly referring to order complexes. This was also done by Provan, who showed that all finite distributive lattices satisfy the Hirsch conjecture [12, p. 62]; a result which we shall now extend.

TheOREM 6.4. Let $P$ be a bounded, locally semimodular finite poset. Then $P$ satisfies the Hirsch conjecture.

Proof. The inequality (6.3) is trivially satisfied if $P$ has length equal to one. We continue by induction on the length of $P$, which of course equals the dimension of the order complex $\Delta(P)$. Assume that length $(P)=\delta>2$ and let $\mathrm{m}: \hat{0}=x_{0} \prec x_{1}$ $\prec \cdots \prec x_{\delta}=\hat{1}$ and $\mathbf{m}^{\prime}: \hat{0}=y_{0} \prec y_{1} \prec \cdots \prec y_{\delta}=\hat{1}$ be two distinct maximal chains in $P$. Also, let $d$ be the greatest integer such that $x_{i}=y_{i}$ for $i=0,1, \ldots, d$, and let $g$ be the least integer for which $y_{d+1}<x_{g}$. Using local semimodularity, select elements $z_{d+2}, z_{d+3}, \ldots, z_{g-1}$ in $P$ as illustrated in Figure 5 above. The interval $\left[y_{d+1}, \hat{1}\right]$ in $P$ is locally semimodular and of length $\delta-d-1<\delta$. By the induction assumption, therefore, there is a path in $\Phi_{\Delta\left(y_{d+1}, 1\right)}$ of length at most $\nu^{\prime}-(\delta-d-1)-1, \nu^{\prime}=\left|\left[y_{d+1}, \hat{1}\right]\right|$, consisting of maximal chains of $\left[y_{d+1}, \hat{1}\right]$, which connects $y_{d+1} \prec y_{d+2} \prec \cdots \prec y_{\delta}=\hat{1}$ to $y_{d+1} \prec z_{d+2} \prec z_{d+3} \prec \cdots \prec$ $z_{g-1} \prec x_{g} \prec x_{g+1} \prec \cdots \prec x_{\delta}=\hat{1}$. If these chains are concatenated with $\hat{0}=y_{0}$ $\prec y_{1} \prec \cdots \prec y_{d}$ we get a path in $\Phi_{\Delta(P)}$ of length $<\nu^{\prime}+d-\delta$ connecting $\mathbf{m}^{\prime}$ to $\mathbf{m}^{\prime \prime}: \quad \hat{0}=y_{0} \prec y_{1} \prec \cdots \prec y_{d+1} \prec z_{d+2} \prec z_{d+3} \prec \cdots \prec z_{g-1} \prec x_{g} \prec x_{g+1}$ $\prec \cdots \prec x_{\delta}=\hat{1}$. It is clear upon inspection of Figure 5 that there is a path in $\Phi_{\Delta(P)}$ of length $g-d-1$ connecting $\mathbf{m}^{\prime \prime}$ to $\mathbf{m}$. Hence, there is a path in $\Phi_{\Delta(P)}$ of length $<\left(\nu^{\prime}+d-\delta\right)+(g-d-1)=\nu^{\prime}+g-\delta-1$ connecting $\mathbf{m}^{\prime}$ to $\mathbf{m}$. Since $x_{i} \notin\left[y_{d+1}, \hat{1}\right]$ for $i=0,1, \ldots, g-1$ we must conclude that $\nu^{\prime}+g\langle\nu=$ $|P|$. Consequently, diam $\Phi_{\Delta(P)} \leqslant \nu^{\prime}+g-\delta-1<\nu-\delta-1$ as required.

A bounded semimodular poset need not satisfy the Hirsch conjecture, as the poset $P$ of Figure 6a shows. $P$ is semimodular but not Cohen-Macaulay and the distance between the two maximal chains through the point $x$ is 6 . However, it is a simple exercise to show that any bounded semimodular poset $P$ of finite length $\delta$ satisfies diam $\Phi_{\Delta(P)}<\delta(\delta-1) / 2$. Hence, we can improve Theorem 6.4 by asserting that for any bounded, locally semimodular finite poset 


$$
\operatorname{diam} \Phi_{\Delta(P)} \leqslant \min \left(\nu-\delta-1, \frac{\delta(\delta-1)}{2}\right) .
$$

This upper bound is, in fact, sharp. For any $\delta>1$ and $\nu>\delta+1$ it is possible, as suggested in Figure $6 \mathrm{~b}$, to construct an upper-semimodular lattice of length $\delta$ and cardinality $\nu$ which achieves equality in (6.5).

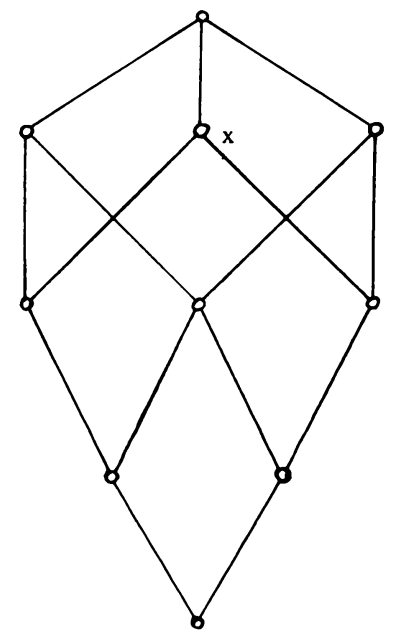

(a)
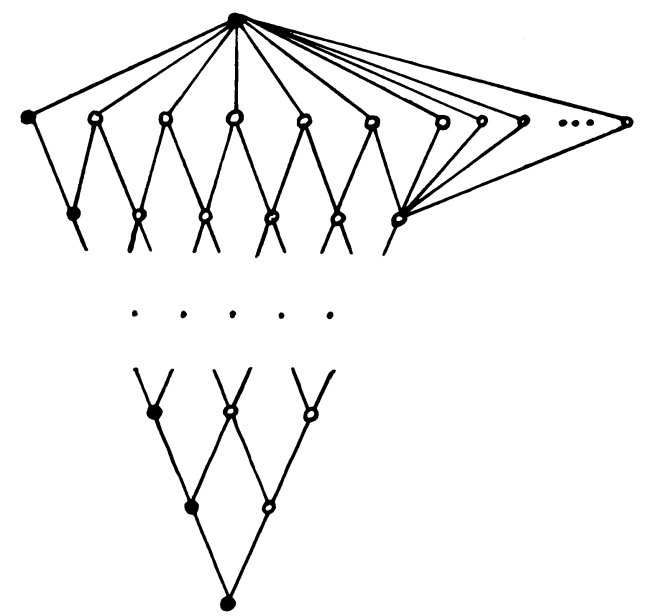

(b)

FIGURE 6

7. Higher order complexes. For a finite poset $P$ let $\Delta_{k}(P), k>1$, denote the simplicial complex of all subsets of $P$ which contain no $(k+1)$-element antichain (totally unordered subset). Thus, for $k=1$, this definition gives us the usual order complex $\Delta(P)$. The higher order complexes $\Delta_{k}(P)$ were suggested to the author by R. Stanley in private correspondence. Guided by analogy with results in the Schubert calculus, Stanley was led to conjecture that the complexes $\Delta_{k}(L)$ are Cohen-Macaulay for all $k \geqslant 1$ when $L$ is a finite planar distributive lattice. In this section we prove a slightly stronger result.

THEOREM 7.1. Let $L$ be a finite planar distributive lattice. Then $\Delta_{k}(L)$ is shellable for all $k \geqslant 1$.

Proof. Let $P$ denote the poset of join-irreducibles of such a lattice $L$ and for $x \in L$ let $I^{x}=\{p \in P \mid p<x\}$. According to Birkhoff's representation theorem for finite distributive lattices [2, Theorem 3, p. 59] the map $x \mapsto I^{x}$ is an isomorphism between $L$ and the poset of order ideals of $P$ ordered by inclusion. Since $L$ is planar, $\boldsymbol{P}$ cannot contain a 3-element antichain. Hence, by Dilworth's well-known decomposition theorem $P$ can be partitioned into two chains. Now, let $Q$ and $R$ be two chains in $P$ such that $P=Q \cup R, Q \cap R=\varnothing$, and for every order ideal $I$ in $P$ write $I_{Q}=I \cap Q$ and $I_{R}=I \cap R$. We can then define a map $d: L \rightarrow \mathbf{R}^{2}$ by $d(x)=\left(\left|I_{R}^{x}\right|-\left|I_{Q}^{x}\right|,\left|I^{x}\right|\right)$. Connect points $d(x)$ and $d(y)$ such that $x$ covers $y$ by straight line segments. It is easy to verify that the graph $\mathscr{D}$ thus drawn in the 
Euclidean plane $\mathbf{R}^{2}$ is a planar embedding of the Hasse diagram of $L$ (we think of the $x$-axis as being "horizontal" and the $y$-axis as pointing "upward"). Two elements $x$ and $y$ of $L$ satisfy $x \leqslant y$ if and only if $I_{Q}^{x} \subseteq I_{Q}^{y}$ and $I_{R}^{x} \subseteq I_{R}^{y}$. Let us define another partial order " $\perp$ " on $L$ by $x<^{\perp} y$ if and only if $I_{Q}^{x} \supseteq I_{Q}^{y}$ and $I_{R}^{x} \subseteq I_{R}^{y}$. It is useful to observe that any pair of elements of $L$ is comparable either under " $<$ " or under " $\leqslant \perp$ ". Thus, an antichain under " $<$ " is a chain under " $<\perp$ ". Further, it is evident that the $\perp$-order is represented by the diagram $\mathscr{D}^{\perp}$ which we get by rotating $\mathscr{D} 90$ degrees counterclockwise in $\mathbf{R}^{2}$. Hence, it makes sense to say that $x$ is "to the left of" $y$ or $y$ is "to the right of" $x$ if and only if $x<{ }^{\perp} y$.

Denote the rank-levels of $L$ by $L_{j}, j=0,1, \ldots, n=|P|$. Thus, $L_{j}=\{x \in$ $L \mid \rho(x)=j\}=\left\{x \in L|| I^{x} \mid=j\right\}$ and so the elements of $L_{j}$ lie on the line $y=j$ in the diagram $\mathscr{D} \subseteq \mathbf{R}^{2}$. Let us say that a sequence $\left(\mathbf{m}_{1}, \mathbf{m}_{2}, \ldots, \mathbf{m}_{k}\right)$ of maximal chains of $L$ is an almost disjoint $k$-sequence of maximal chains (a.d. $k$-sequence, for short) if for every $j=0,1, \ldots, n$,

(i) $\mathbf{m}_{i} \cap L_{j}$ is strictly to the left of $\mathbf{m}_{i+1} \cap L_{j}$ for $i=1,2, \ldots,\left|L_{j}\right|-1$, and

(ii) $\mathbf{m}_{i} \cap L_{j}=\mathbf{m}_{i+1} \cap L_{j}$ for $i=\left|L_{j}\right|,\left|L_{j}\right|+1, \ldots, k-1$.

Note that the definitions of the diagram $\mathscr{D}$, the order " $\perp$ " and, hence, of an a.d. $k$-sequence depend on the chosen decomposition of $P$ into chains $Q$ and $R$. However, for any such choice we can now identify the facets of $\Delta_{k}(L)$.

LEMMA. Let $L$ be a finite planar distributive lattice and decompose the join-irreducibles $P$ into chains $Q$ and $R$.

(a) If $F \subseteq L$ does not contain a $(k+1)$-element antichain then $F$ is contained in the union of an almost disjoint $k$-sequence of maximal chains of $L$.

(b) The map $\left(\mathbf{m}_{1}, \mathbf{m}_{2}, \ldots, \mathbf{m}_{k}\right) \stackrel{\varphi}{\mapsto} \cup_{i=1}^{k} \mathbf{m}_{i}$ is a bijection between the set of a.d. $k$-sequences of maximal chains of $L$ and the set of facets of $\Delta_{k}(L)$.

Proof. The lemma holds trivially for $k=1$. We shall prove part (a) for $k>2$ by induction on the order $|L|$. The argument is based on an idea of J. Backelin's. Statement (a) is trivially true for all $k \geqslant 1$ when $|L|=2$. Assume that (a) has been proven for all $k \geqslant 1$ and for all lattices of order less than that of a given lattice $L$. If there is a singleton rank-level $L_{j}=\{x\}$ with $0<j<n=|P|$, then the induction hypothesis can be applied separately in the two intervals $[\hat{0}, x]$ and $[x, \hat{1}]$, and respective members of the two a.d. $k$-sequences can be glued together at $x$ to form an a.d. $k$-sequence in $L$. We may therefore assume that $\left|L_{j}\right| \geqslant 2$ for $j=1,2, \ldots$, $n-1$. Denote by $A$ the set of elements in $L$ which are join-irreducible and meet-irreducible and lie on the left boundary of the diagram $\mathscr{D}$. It is easy to see that $A$ is not empty, in fact $A$ coincides with the set of minimal elements of the diagram $\mathscr{D}^{\perp}$. Now, consider a subset $F$ of $L$ which does not contain a $(k+1)$ antichain, $k \geqslant 2$. In case $A \notin F$ then $F \subseteq L-\{a\}$ for some $a \in A$. Since $a$ is doubly irreducible, $L-\{a\}$ is a sublattice of $L$, hence distributive, and the graph $\mathscr{D}-d(a)$ is a planar embedding of the Hasse diagram of $L-\{a\}$ in the prescribed sense. So by the induction assumption $F \subseteq \cup_{i=1}^{k} \mathbf{m}_{i}$, where $\left(\mathbf{m}_{1}, \mathbf{m}_{2}, \ldots, \mathbf{m}_{k}\right)$ is an a.d. $k$-sequence of maximal chains of $L-\{a\}$. Since the 
rank-levels $L_{j-1}, L_{j}$ and $L_{j+1}$, where $a \in L_{j}$, are related as illustrated in Figure 7, it is obvious how to derive from $\left(\mathbf{m}_{1}, \mathbf{m}_{2}, \ldots, \mathbf{m}_{k}\right)$ an a.d. $k$-sequence of maximal chains of $L$ which covers $F$.
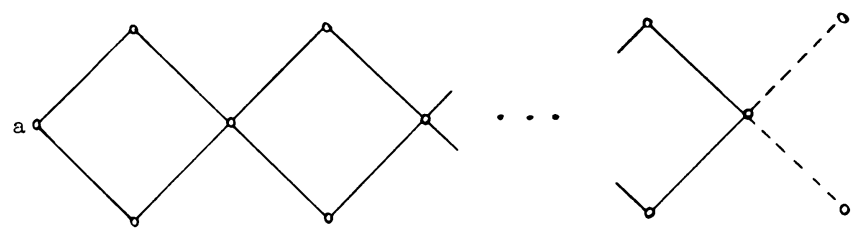

FIGURE 7

Next we must consider the case when $A \subseteq F$. Let $\mathrm{m}_{0}$ denote the maximal chain of $L$ which lies on the left boundary of $\mathscr{D}$. If $B=\mathrm{m}_{0}-\{\hat{0}, \hat{1}\}$, then $A \subseteq B$, and $L-B$ is a sublattice of $L$. Arguing as above, it is clear that the induction assumption applies to $L-B$. Suppose that $F-B$ contains a $k$-element antichain $C$. As observed earlier, $C$ is then a chain under the $\perp$-order of $L$. Let $x$ be the $\perp$-least element of $C$, and let $y$ be the $\perp$-least (or furthest left) element of $L_{j}$, where $x \in L_{j}$. Then $y \in B$ and since $x \in F-B$ we have $y<^{\perp} x$. Let $z$ be a $\perp$-minimal element of $L$ with $z<^{\perp} y$. Then $z \in A$, and $C \cup\{z\}$ is a $(k+1)$ element antichain which is contained in $C \cup A \subseteq F$. This contradiction shows that $F-B$ cannot contain a $k$-element antichain. By the induction assumption there is therefore an a.d. $(k-1)$-sequence $\left(\mathbf{m}_{1}, \mathbf{m}_{2}, \ldots, \mathbf{m}_{k-1}\right)$ of maximal chains of $L-$ $B$ such that $F-B \subseteq \cup{ }_{i=1}^{k-1} \mathbf{m}_{i}$. But then $\left(\mathbf{m}_{0}, \mathbf{m}_{1}, \ldots, \mathbf{m}_{k-1}\right)$ is an a.d. $k$-sequence of maximal chains of $L$ such that $F \subseteq \bigcup_{\phi}{ }_{i=0}^{k-1} \mathbf{m}_{i}$. Thus, part (a) is proved.

Consider the map $\left(\mathbf{m}_{1}, \mathbf{m}_{2}, \ldots, \mathbf{m}_{k}\right) \stackrel{\oplus}{\mapsto} \cup_{i=1}^{k} \mathbf{m}_{i}$ of part (b). A subset of $L$ of the form $\cup_{i=1}^{k} \mathbf{m}_{i}$ obviously cannot contain a $(k+1)$-element antichain. Also, it is maximal with this property, because if $x \in L-S$ where $S=\cup_{i=1}^{k} \mathbf{m}_{i}$ and $\left(\mathbf{m}_{1}, \mathbf{m}_{2}, \ldots, \mathbf{m}_{k}\right)$ is an a.d. $k$-sequence, then $x \in L_{j}$ for a $j$ such that $\left|L_{j}\right|>k$, and $\left(S \cap L_{j}\right) \cup\{x\}$ is a $(k+1)$-element antichain contained in $S \cup\{x\}$. The map $\varphi$ is clearly injective, and surjectivity was established in part (a) above.

Having thus characterized the facets of $\Delta_{k}(L)$, we are now in a position to conclude the proof of Theorem 7.1 by assigning a shelling order. For a given finite planar distributive lattice $L$, decompose its poset of join-irreducibles $\boldsymbol{P}$ into chains $Q$ and $R$ and draw the corresponding diagram $\mathscr{D}$ in $\mathbf{R}^{2}$ as explained above. Let $\omega$ : $P \rightarrow[n], n=|P|$, be the unique order-preserving bijection which for all $q \in Q$ and $r \in R$ satisfies $\omega(q)>\omega(r)$ only if $q>r$. Then $\omega$ is an admissible map and induces an $L$-labeling $\gamma$ of $L$ (cf. §3). The $L$-labeling $\gamma$ has the distinguishing feature that the unique rising unrefinable chain in any interval $[x, y]$ follows the left boundary of $[x, y]$ as drawn in $\mathscr{D}$. If $F$ is a facet of $\Delta_{k}(L)$ and $\left(\mathbf{m}_{1}, \mathbf{m}_{2}, \ldots, \mathbf{m}_{k}\right)$ is the unique a.d. $k$-sequence of maximal chains such that $F=\cup{ }_{i=1}^{k} \mathbf{m}_{i}$, then associate with $F$ the $n k$-tuple $\sigma(F)=\left(\pi\left(\mathbf{m}_{1}\right), \pi\left(\mathbf{m}_{2}\right), \ldots, \pi\left(\mathbf{m}_{k}\right)\right) \in \mathbf{P}^{n k}$, where $\pi\left(\mathbf{m}_{i}\right)$ is the usual Jordan-Hölder sequence of the maximal chain $\mathbf{m}_{i}$ under the edge-labeling $\gamma$ (cf. $\$ 1$ ). $F \neq F^{\prime}$ implies that $\sigma(F) \neq \sigma\left(F^{\prime}\right)$, and the lexicographic order of the $n k$-tuples $\sigma(F)$ in $P^{n k}$ determines a linear order $\Omega$ of the facets of $\Delta_{k}(L)$. It remains to verify that $\Omega$ is a shelling. 
Let $\left(\mathbf{m}_{1}, \mathbf{m}_{2}, \ldots, \mathbf{m}_{k}\right)$ and $\left(\mathbf{m}_{1}^{\prime}, \mathbf{m}_{2}^{\prime}, \ldots, \mathbf{m}_{k}^{\prime}\right)$ be two a.d. $k$-sequences of maximal chains of $L$ and let $F=\cup_{i=1}^{k} \mathbf{m}_{i}$ and $F^{\prime}=\cup_{i=1}^{k} \mathbf{m}_{i}^{\prime}$. Suppose that $F^{\prime}<{ }^{\Omega} F$. We may then assume that $\mathbf{m}_{i}=\mathbf{m}_{i}^{\prime}$ for $i=1,2, \ldots, e-1$, and $\mathbf{m}_{e} \neq \mathbf{m}_{e}^{\prime}$. Let $\mathbf{m}_{e}$ : $\hat{0}=x_{0} \prec x_{1} \prec \cdots \prec x_{n}=\hat{1}, \mathbf{m}_{e}^{\prime}: \hat{0}=y_{0} \prec y_{1} \prec \cdots \prec y_{n}=\hat{1}$ and suppose that $x_{i}=y_{i}$ for $i=0,1, \ldots, g, x_{i} \neq y_{i}$ for $i=g+1, g+2, \ldots, h-1$, and $x_{h}=$ $y_{h}$. Then, since $F^{\prime}<^{\Omega} F$, by construction $y_{g+1}$ lies to the left of $x_{g+1}$ in $\mathscr{D}$, and the planarity of $\mathscr{D}$ forces $y_{i}$ to be to the left of $x_{i}$ for $i=g+1, g+2, \ldots, h-1$. The relevant parts of $\mathscr{D}$ are illustrated in Figure 8.

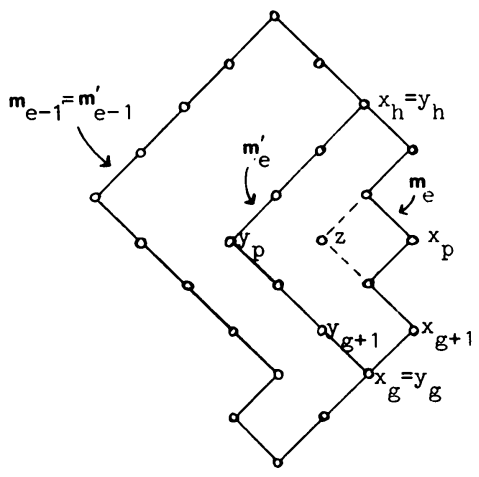

FIGURE 8

The chain $x_{g} \prec x_{g+1} \prec \cdots \prec x_{h}$ cannot be rising, since then it would by construction trace the left boundary of the interval $\left[x_{g}, x_{h}\right]$. Say there is a descent $\gamma\left(x_{p-1}, x_{p}\right)>\gamma\left(x_{p}, x_{p+1}\right)$ at $p, g<p<h$. Then there exists a unique $z \in L$ such that $x_{p-1} \prec z \prec x_{p+1}$ is rising. By our construction $z$ lies immediately to the left of $x_{p}$ in the rank-level $L_{p}$, hence to the right (not necessarily strictly) of $y_{p}$. Let $\mathbf{m}_{z}$ be the maximal chain which we get by replacing $x_{p}$ by $z$ in $\mathbf{m}_{e}$. It is clear from the geometric situation (with $z$ in between $y_{p}$ and $x_{p}$ in $L_{p}$ ) that $\left(\mathbf{m}_{1}, \mathbf{m}_{2}, \ldots, \mathbf{m}_{e-1}, \mathbf{m}_{z}, \mathbf{m}_{e+1}, \mathbf{m}_{e+2}, \ldots, \mathbf{m}_{k}\right)$ is an a.d. $k$-sequence of maximal chains. Let $F^{\prime \prime}$ be the corresponding facet of $\Delta_{k}(L)$. Then $F^{\prime \prime}<^{\Omega} F$ and $F^{\prime} \cap F \subseteq$ $F^{\prime \prime} \cap F=F-\left\{x_{p}\right\}$. Hence, $\Omega$ is a shelling.

There seems to be no immediate generalization to the above theorem. For instance, consider the planar modular lattice $M$ and the 3-dimensional distributive lattice $D$ of Figure 9.

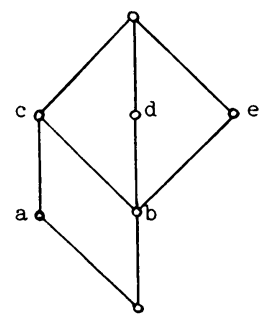

M

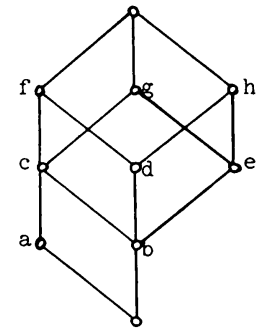

D

FIgURE 9 
The complexes $\Delta_{2}(M)$ and $\Delta_{2}(D)$ are not Cohen-Macaulay, in fact, they are not even pure (e.g. $\{\hat{0}, a, b, c, d, \hat{1}\}$ and $\{\hat{0}, b, d, e, \hat{1}\}$ are facets of $\Delta_{2}(M)$ and $\{\hat{0}, a, b, c, d, g, h, \hat{\mathbf{1}}\}$ and $\{\hat{0}, b, d, e, f, g, \hat{1}\}$ are facets of $\left.\Delta_{2}(D)\right)$.

Appendix. We shall briefly review the definitions of shellable and CohenMacaulay complexes and also comment on the logical relationship of these and related concepts.

A finite simplicial complex $\Delta$ is by definition a nonvoid family of subsets, called simplices or faces, of a finite set $V$, called the vertex-set, such that $v \in V$ implies $\{v\} \in \Delta$ and $F \subseteq G \in \Delta$ implies $F \in \Delta$. In particular, $\varnothing \in \Delta$. The dimension of a face $F, \operatorname{dim} F$, is $|F|-1$, and the dimension of $\Delta, \operatorname{dim} \Delta$, is $\max \{\operatorname{dim} F \mid F \in \Delta\}$. The faces which are maximal under inclusion are called facets. A complex is pure if all its facets are equicardinal.

Let $\Delta$ be a finite simplicial complex. We say that $\Delta$ is shellable if $\Delta$ is pure and the facets of $\Delta$ can be given a linear order $F_{1}, F_{2}, \ldots, F_{t}$ in such a way that:

if $1 \leqslant i<k \leqslant t$ then there is a $j, 1 \leqslant j<k$, and an $x \in F_{k}$ such that $F_{i} \cap F_{k} \subseteq F_{j} \cap F_{k}=F_{k}-\{x\}$.

In other words, the facet $F_{k}$ is required to intersect the complex $\cup_{i=1}^{k-1} F_{i}$ in a nonempty union of maximal proper faces of $F_{k}$. A linear order of the facets which satisfies this requirement is called a shelling. Shellability has been most intensively investigated for complexes which triangulate spheres and balls (see the survey [4]).

For a simplex $F$ in a complex $\Delta$ the link of $F$ is the subcomplex lk $F=\{G \in$ $\Delta \mid G \cup F \in \Delta, G \cap F=\varnothing\}$. Let $R$ denote the ring of rational integers $\mathbf{Z}$ or a field $k$, and let $\tilde{H}_{*}(\Delta, R)$ denote reduced simplicial homology of $\Delta$ with coefficients in $R$. A simplicial complex $\Delta$ is said to be Cohen-Macaulay over $R$ if $\tilde{H}_{i}(\mathrm{lk} F, R)=0$ for all $F \in \Delta$ and $i<\operatorname{dim}(\mathrm{lk} F)$. The motivation for this terminology is to be found in a theorem of G. Reisner [15]. Let $V=\left\{x_{1}, x_{2}, \ldots, x_{n}\right\}$ be the vertex-set of $\Delta$ and define $I_{\Delta}$ to be the ideal in the polynomial ring $R\left[x_{1}, x_{2}, \ldots, x_{n}\right]$ generated by all square-free monomials $x_{i_{1}} x_{i_{2}} \cdots x_{i_{e}}$ such that $\left\{x_{i_{1}}, x_{i_{2}}, \ldots, x_{i_{e}}\right\} \notin$ $\Delta$. Reisner's theorem states that the complex $\Delta$ is Cohen-Macaulay over $R$ if and only if $R\left[x_{1}, x_{2}, \ldots, x_{n}\right] / I_{\Delta}$ is a Cohen-Macaulay ring. Using the Universal Coefficient Theorem one can verify that $\Delta$ is Cohen-Macaulay over $\mathbf{Z}$ if and only if $\Delta$ is Cohen-Macaulay over all fields, and if $\Delta$ is Cohen-Macaulay over some field then $\Delta$ is Cohen-Macaulay over the rationals $\mathbf{Q}$. Also, it is easily shown that a Cohen-Macaulay complex (over any $R$ ) must be pure. For more information about Cohen-Macaulay complexes see the surveys [9] and [20], and also [21].

The following variation of the Cohen-Macaulay concept was introduced by $D$. Quillen in [14]: a pure complex $\Delta$ is said to be homotopy Cohen-Macaulay if the homotopy groups $\pi_{i}(\mathrm{lk} F)$ are trivial for all $F \in \Delta$ and $i<\operatorname{dim}(\mathrm{lk} F)$. In view of the Hurewicz isomorphism theorem it is clear that $\Delta$ is homotopy Cohen-Macaulay if and only if $\Delta$ is Cohen-Macaulay over $Z$ and in addition the links $\mathbf{l k} F$ are simply-connected for all faces $F \in \Delta \operatorname{such}$ that $\operatorname{dim} F<\operatorname{dim} \Delta-3$.

Following Hochster [8] and Stanley [20] we call a complex constructible if it belongs to the class of complexes defined recursively by: (1) a simplex is construct- 
ible, and (2) if $\Delta_{1}, \Delta_{2}$ and $\Delta_{1} \cap \Delta_{2}$ are constructible and $\operatorname{dim} \Delta_{1}=\operatorname{dim} \Delta_{2}=\operatorname{dim}\left(\Delta_{1}\right.$ $\left.\cap \Delta_{2}\right)+1$, then $\Delta_{1} \cup \Delta_{2}$ is constructible. Clearly, a shellable complex is constructible. Also, using the Mayer-Vietoris exact sequence for homology and the van Kampen theorem for fundamental groups one can verify that a constructible complex must be homotopy Cohen-Macaulay (cf. [8, Remark 8]).

Summarizing, we have seen that among the properties "shellable", "constructible", "homotopy Cohen-Macaulay" and "Cohen-Macaulay over $\mathbf{Z}$ " of a simplicial complex, each implies its successor. Counterexamples are known to two of the three converse implications. R. D. Edwards' 5-sphere, which is the double suspension of a non-simply-connected homology 3-sphere (see [4, p. 41]), is CohenMacaulay over $\mathbf{Z}$ but not homotopy Cohen-Macaulay. Also, M. E. Rudin's unshellable 3-ball (see [4, p. 40]) has been proved constructible by S. Provan [13]. We do not know of any complex which is homotopy Cohen-Macaulay but not constructible, although it seems likely that such examples should exist.

\section{REFERENCES}

1. K. Baclawski, Homology and combinatorics of ordered sets, Thesis, Harvard Univ., Cambridge, Mass., 1976.

2. G. Birkhoff, Lattice theory, 3rd ed., Amer. Math. Soc. Colloq. Publ., vol. 25, Amer. Math. Soc., Providence, R. I., 1967.

3. H. Bruggesser and P. Mani, Shellable decompositions of cells and spheres, Math. Scand. 29 (1971), 197-205.

4. G. Danaraj and V. Klee, Which spheres are shellable?, Ann. Discrete Math. 2 (1978), 33-52.

5. G. Ewald and G. C. Shephard, Stellar subdivisions of boundary complexes of convex polytopes, Math. Ann. 210 (1974), 7-16.

6. F. Farmer, Cellular homology for posets, 1st ed., Arizona State Univ. (preprint).

7. J. Folkman, The homology groups of a lattice, J. Math. Mech. 15 (1966), 631-636.

8. M. Hochster, Rings of invariants of tori, Cohen-Macaulay rings generated by monomials, and polytopes, Ann. of Math. (2) 96 (1972), 318-337.

9. Cohen-Macaulay rings, combinatorics, and simplicial complexes, Proc. 2nd Oklahoma Ring

Theory Conf. (B. McDonald and R. Morris, ed.), Dekker, New York, 1977, pp. 171-223.

10. G. Kreweras, Sur les partitions non croisées d'un cycle, Discrete Math. 1 (1972), 333-350.

11. B. Lindström, Problem P73, Aequationes Math. 6 (1971), 113.

12. S. Provan, Decompositions, shellings, and diameters of simplicial complexes and convex polyhedra, Thesis, Cornell Univ., Ithaca, N. Y., 1977.

13.

14. D. Quillen, Homotopy properties of the poset of non-trivial p-subgroups of a group, Advances in Math. 28 (1978), 101-128.

15. G. Reisner, Cohen-Macaulay quotients of polynomial rings, Advances in Math. 21 (1976), 30-49.

16. I. Rival, A note on linear extensions of irreducible elements in a finite lattice, Algebra Universalis 6 (1976), 99-103.

17. G.-C. Rota, On the foundations of Combinatorial Theory I: Theory of Möbius functions, $Z$. Wahrscheinlichkeitstheorie und Verw. Gebiete 2 (1964), 340-368.

18. R. Stanley, Supersolvable lattices, Algebra Universalis 2 (1972), 197-217.

19. ___ Finite lattices and Jordan-Hölder sets, Algebra Universalis 4 (1974), 361-371.

20. Cohen-Macaulay complexes, Higher Combinatorics (M. Aigner, ed.), Reidel, Dordrecht and Boston, Mass., 1977, pp. 51-62.

21. , Balanced Cohen-Macaulay complexes, Trans. Amer. Math. Soc. 249 (1979), 139-157.

22. M. Suzuki, Structure of a group and the structure of its lattice of subgroups, Springer-Verlag, Berlin and New York, 1956.

23. K. Baclawski, Cohen-Macaulay ordered sets, J. Algebra (to appear).

24. Cohen-Macaulay connectivity of geometric lattices, Haverford College, 1979 (preprint).

Department of Mathematics, University of Stockholm, Box 6701, S-11385 Stockholm, SWEDEN 